\title{
Wavelet-based multi-resolution statistics for optical imaging signals: Application to automated detection of odour activated glomeruli in the mouse olfactory bulb
}

\author{
Brice Bathellier, ${ }^{\mathrm{a}, \mathrm{b}}$ Dimitri Van De Ville, ${ }^{\mathrm{c}}$ Thierry Blu, ${ }^{\mathrm{c}}$ Michael Unser, ${ }^{\mathrm{c}}$ and Alan Carleton ${ }^{\mathrm{a}, *}$ \\ ${ }^{a}$ Flavour Perception Group, Brain Mind Institute, Ecole Polytechnique Fédérale de Lausanne, (EPFL), CH-1015, Switzerland \\ ${ }^{\mathrm{b}}$ Laboratory of Computational Neuroscience, Brain Mind Institute, Ecole Polytechnique Fédérale de Lausanne, (EPFL), CH-1015, Switzerland \\ 'Biomedical Imaging Group, Ecole Polytechnique Fédérale de Lausanne (EPFL), CH-1015, Switzerland
}

Received 17 August 2006; revised 4 October 2006; accepted 26 October 2006

Available online 19 December 2006

\begin{abstract}
Optical imaging techniques offer powerful solutions to capture brain networks processing in animals, especially when activity is distributed in functionally distinct spatial domains. Despite the progress in imaging techniques, the standard analysis procedures and statistical assessments for this type of data are still limited. In this paper, we perform two in vivo non-invasive optical recording techniques in the mouse olfactory bulb, using a genetically expressed activity reporter fluorescent protein (synaptopHfluorin) and intrinsic signals of the brain. For both imaging techniques, we show that the odour-triggered signals can be accurately parameterized using linear models. Fitting the models allows us to extract odour specific signals with a reduced level of noise compared to standard methods. In addition, the models serve to evaluate statistical significance, using a wavelet-based framework that exploits spatial correlation at different scales. We propose an extension of this framework to extract activation patterns at specific wavelet scales. This method is especially interesting to detect the odour inputs that segregate on the olfactory bulb in small spherical structures called glomeruli. Interestingly, with proper selection of wavelet scales, we can isolate significantly activated glomeruli and thus determine the odour map in an automated manner. Comparison against manual detection of glomeruli shows the high accuracy of the proposed method. Therefore, beyond the advantageous alternative to the existing treatments of optical imaging signals in general, our framework propose an interesting procedure to dissect brain activation patterns on multiple scales with statistical control.

(c) 2006 Elsevier Inc. All rights reserved.
\end{abstract}

Keywords: Wavelet transform; Intrinsic imaging; Glomeruli; SynaptopHfluorin; Olfaction

Abbreviations: OB, olfactory bulb; LM, linear model; DWT, discrete wavelet transform; S.D., standard deviation; $\mathrm{SpH}$, SynaptopHfluorin.

* Corresponding author. Fax: +41 216931650.

E-mail address: alan.carleton@epfl.ch (A. Carleton).

Available online on ScienceDirect (www.sciencedirect.com).

\section{Introduction}

In recent years, optical imaging of brain signals has become an undeniable technique in neuroscience research. An important reason of this success is due to its capability to observe neuronal activity at many points in space simultaneously, and this in vivo (or in vitro) at spatial levels ranging from cellular to network scales (c.f. Grinvald and Hildesheim, 2004). For example, optical imaging permitted to visualize ensembles of cells sharing the same function inside various sensory areas of the mammalian brain (Grinvald and Hildesheim, 2004), but it was also used (although in a different context) to record membrane potential in the entire dendritic arborisation of a single neuron (Djurisic et al., 2004). Such a broad range of possibilities cannot to be expected from classical electrophysiology. Indeed, while electrophysiology is relying on electrical signals, optical imaging uses reporter signals that can be linked to activity in many different ways. Most famous examples of reporter signals are probably dye molecules like voltage or calcium sensitive dyes, which give a very direct coupling with neuronal activity. Their drawback is invasiveness, as they have to be applied onto the brain and to penetrate the neuron membrane. Therefore, non-invasive methods based on intrinsic modification of the tissue upon activation have offered a good complement to dye experiments. In general, these signals are based on rather indirect (metabolic) responses, but require no tissue manipulation for observation. Lately, the possibility of a compromise between non-invasiveness and signal specificity has emerged. Promising results have been obtained with genetically modified animals that express reporter fluorescent proteins in targeted neuronal populations (Bozza et al., 2004; Hasan et al., 2004), which then can be observed optically with very little preparation even in vivo.

Despite the strong technical development and diversification of the imaging techniques, little has been done yet to improve specifically the treatment and analysis of optical recordings. Signals of interest are generally extracted by subtraction (or 
division) of a reference (so-called "blank") image or image time series. The latter method has the advantage of simplicity. However, some dyes show strong photobleaching or phototoxicity, and in such case it can be an experimental limitation to spend illumination time for acquiring the blank image sequence. Moreover, blank subtraction is sensitive with respect to noise because it simply adds up pixel variability of the "blank" and "signal" recordings. Finally, the determination of regions activated by a particular stimulus is usually based on manual selection or arbitrary thresholds rather than on statistical methods.

In this study, we develop a combination of linear model (LM) fitting and wavelet-based multi-resolution statistical analysis as tools for optical imaging processing. We apply these methods to the imaging of odour evoked activity patterns in the mouse olfactory bulb. In this context, we first show that LM fitting permits to extract relevant signals without the standard "blank" subtraction and with better noise suppression. In a second part, we use the LM analysis for statistical assessment of odour-dependant responses. Since a pixel-by-pixel analysis does not exploit the spatial correlation available in the data, we deploy a recent framework based on the spatial wavelet transform (Van De Ville et al., 2004). Moreover, the multi-resolution decomposition by the wavelet transform is particularly interesting in this application, because it allows us to select spatial activation patterns of an appropriate resolution range. This way, we can specifically detect and assess the activated olfactory bulb glomeruli, which are the meaningful units of the odour code at the entry of the olfactory bulb.

\section{Materials and methods}

Experimental setup

Animals and surgical procedures

SynaptopHfluorin $(\mathrm{SpH})$ and intrinsic signal experiments were performed on adults (8-12 weeks) B6.129P2-Omp ${ }^{\text {tm2(spH)Mom/J }}$ mice (Bozza et al., 2004) available from The Jackson Laboratory (Bar Harbor, Maine). In addition, for intrinsic signal some adult C57B16J mice were also used. Animals were anesthetized with urethane $(1.5 \mathrm{~g} / \mathrm{kg}$, i.p.). Heart beat, respiration rate and lack of pain reflexes were monitored throughout the experiment. Additional urethane (10-20\% of initial dose) was administered if needed. The body temperature was kept between 36.5 and $37.5^{\circ} \mathrm{C}$ using a heating pad and a rectal probe (FHC, Bowdoinham, ME). A local anaesthetic, lidocaine (Streuli, Uznach, Switzerland), was injected before any skin cut. For imaging, the skin was opened above the dorsal part of the olfactory bulb and the bone was thinned to allow good light transmission. All animal protocols conformed to the Swiss federal laws.

\section{Optical imaging}

For intrinsic signal imaging (see also sketch Fig. 1A), the olfactory bulb was illuminated with red light at $700 \mathrm{~nm}$ (BP $20 \mathrm{~nm}$ ) using a stable $100 \mathrm{~W}$ halogen lamp and a light guides system. Images were acquired at $5 \mathrm{~Hz}$ for $10 \mathrm{~s}$ using the Imager $3001 \mathrm{~F}$ system (Optical Imaging, Mountainside, NJ) or alternatively at $25 \mathrm{~Hz}$ using the Micam Ultima system (Brainvision, Tokyo, Japan)
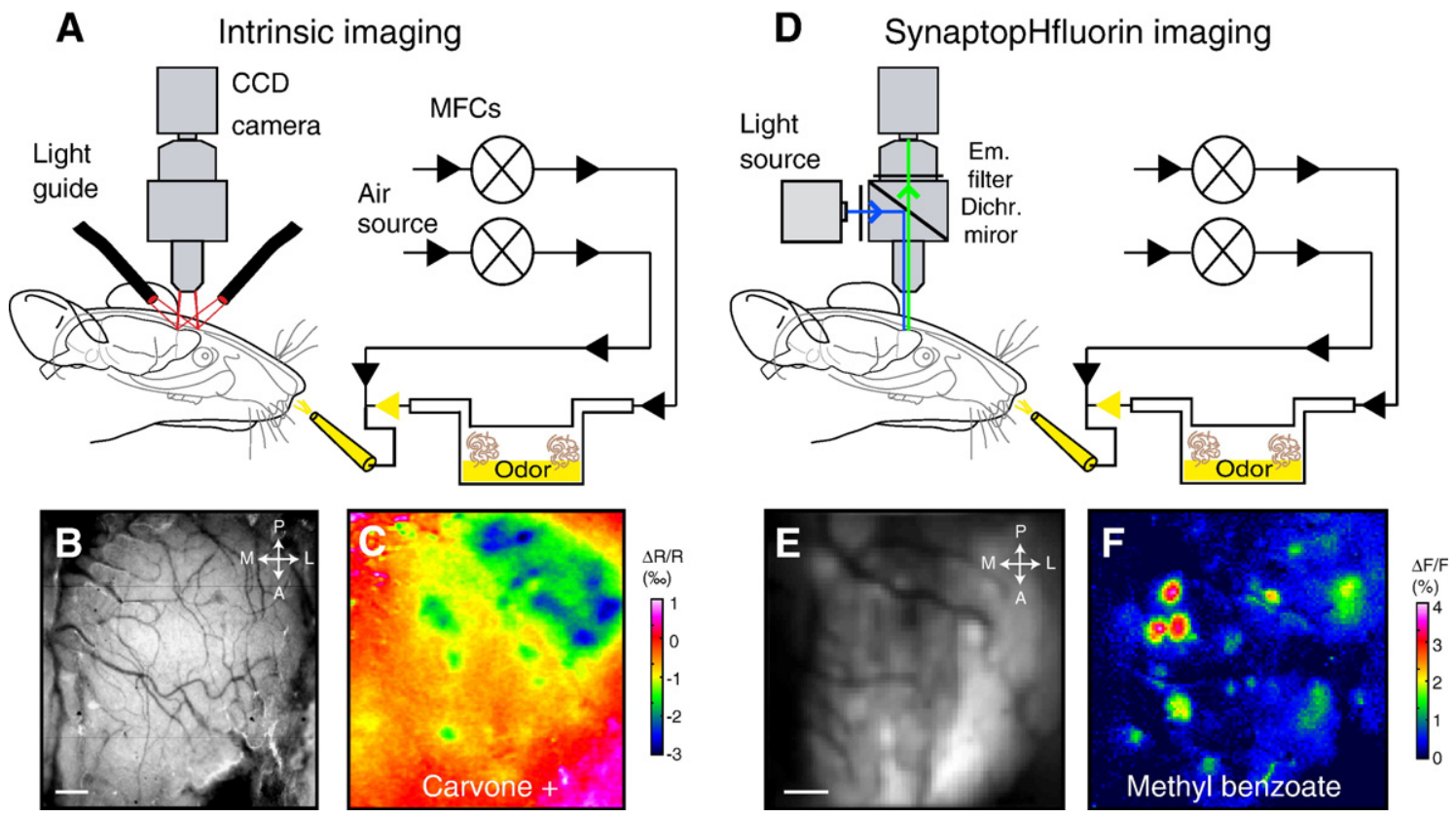

Fig. 1. Intrinsic and synaptopHfluorin imaging of odour activated glomeruli pattern. (A) Sketch of the intrinsic optical imaging setup and odour stimulation apparatus. MFC: Mass Flow Controller. (B) Blood vessel pattern of the dorsal part of the olfactory bulb. Incident green light (546 $\mathrm{nm}$ ) is used to obtain a good contrast. Medial, lateral, anterior, and posterior orientation are indicated by the arrows. (C) Example of an intrinsic imaging odour map obtained in the mouse presented in (B) after delivery of Carvone $+(50 \%)$. Activated glomeruli (green and blue spots) showed a decrease of reflectance after odour stimulation. The map (average over $5 \mathrm{~s}$ following odour stimulation) is revealed by dividing the series of acquired images with the average of $1 \mathrm{~s}$ recording right before stimulation. (D) Schematic representation of the $\mathrm{SpH}$ imaging setup. Excitation, emission, and dichroic filter are represented. (E) Resting fluorescence image for SpH signal. Orientation is the same as for the intrinsic imaging example (different animal is shown). (F) Example of an odour map representing variations of the SpH fluorescent signal after methyl benzoate (10\%) stimulation; these are revealed by subtraction with a blank presentation and normalisation (division) with the first $1 \mathrm{~s}$ of recording averaged image. Scale bars $200 \mu \mathrm{m}$. 
mounted both on a custom build macroscope (Navitar $17 \mathrm{~mm}$ or $25 \mathrm{~mm}$, bottom lenses, Nikon $135 \mathrm{~mm}$, upper lens; total magnification $7.9 \times$ or $5.4 \times$ ). The dimension of the collected pixel matrix was $256 \times 256$ pixels for the Imager $3001 \mathrm{~F}$ system, and $100 \times 100$ for the Micam System. The blood vessel pattern was taken using green light (546 $\mathrm{nm}$ interference filter) at the beginning of each experimental session.

For $\mathrm{SpH}$ imaging (see also sketch Fig. 1D), we used the same macroscope as for intrinsic imaging, but the olfactory bulb (OB) was illuminated with $480 \mathrm{~nm}$ light (filter HQ 480/40), reflected to the brain by a dichroic mirror (Q 505 LP). Emitted light passed through a $535 \mathrm{~nm}$ band pass filter (HQ 535/50). For $\mathrm{SpH}$ recordings only the Micam system was used.

\section{Odour stimulation}

Odours were delivered for $5 \mathrm{~s}$ by a custom build olfactometer $1.5 \mathrm{~s}$ after recording onset (see sketch Fig. 1A). Air was purified with a charcoal filter. The flow was then split into a dilution stream and an odour stream that passed through saturated bottles of odorants. These streams were merged right before the output. Before odour presentation, odour flows were directed for $5 \mathrm{~s}$ into an exhaust circuit placed at the output to ensure that flows have reached a steady state, and were then sent to the nose by switching a valve. Computer driven mass flow controllers (Pneucleus Technologies LLC, Hollis, NH) and electromagnetic valves allowed precise control of odour selection, dilution and timing. The steady state was assessed offline with a mass flow meter (Sierra Instruments Inc, Monterey, CA). The latter procedure was important to obtain a sharp stimulation onset and concentration stability during odour presentation. Further in the text, odour concentration is expressed as a percentage of the saturated vapour pressure, which reflects the relative flow rates of the odour and dilution streams. For example, a value of $5 \%$ means that the saturated odour stream was set at a flow rate 20 times lower than the dilution stream. In all cases, the total output flow rate was equal to $400 \mathrm{sccm}$.

\section{Data handling (standard procedures)}

Recorded image series were analysed with a custom image viewer and scripts running in Matlab (The MathWorks Inc; Natick, MA). For processing the intrinsic signals, the standard treatment consisted in dividing the entire image series by the average frame over the first $1 \mathrm{~s}$ of the recording. For $\mathrm{SpH}$ signals, the response has to be deduced from the photobleaching process. For this, the standard treatment was simply a frame-by-frame subtraction with a "blank" (no stimulus presented) image series. In addition, data normalization was performed by division of the average frame over the first $1 \mathrm{~s}$. Thus pixel values represent the fraction (or percentage) of light amplitude changes corresponding to either reflectance $(\Delta R / R)$ or fluorescence $(\Delta F / F)$ modulations.

Noise amplitude in recordings was evaluated by computing the standard deviation (S.D.) of blank presentations evaluated in time (i.e. S.D. of pixel time-course averaged over all pixels) and space (i.e. S.D. of each frame averaged over time). Variability of odour responses was also quantified by computing the distance between repetitions of the same odour presentation. Variability in time was the average Euclidean distance between pixels time-courses (taken as 250-dimensional vectors). Variability in space was the average Euclidean distance between frames (taken as 10000-dimensional vectors). To be able to compare temporal and spatial analyses, we normalized the distance by the vector length (i.e. the number of frames for time analysis and the number of pixels for space) in each case.

\section{In vivo imaging of odour evoked signals}

In the olfactory system, odorant molecules are detected by olfactory receptors expressed on cilia of sensory neurons located in the olfactory epithelium in the nasal cavity. The sensory neurons project their axon onto a defined anatomical structure named glomerulus (synaptic contact between sensory neurons axon and dendrite of relay neurons) in the olfactory bulb (OB). Two main features define the proposed coding logic of odours (for review, Mombaerts, 2004). First, one sensory neuron expresses only one receptor out of a repertoire of a thousand possible. Second, all the sensory neurons expressing the same receptor project their axon onto the same glomerulus. Therefore understanding the odour coding goes through reliable mapping of responsive glomeruli.

In order to visualize glomeruli patterns, we first conducted intrinsic signal imaging that measures light reflectance modification after neuronal activation (Fig. 1A). The signal originates from different mechanisms such as changes in the physical properties of the tissue and/or changes of fluorescence or absorption of intrinsic molecules (e.g. the haemoglobin, for review, Grinvald et al., 1999). However, all these signals can be efficiently used for functional mapping and are giving similar results (Frostig et al., 1990). Since the signals are intrinsic to the tissue, experiments can be performed through the thinned skull and image focus is done on the blood vessels (Fig. 1B). As shown previously in the rat (Meister and Bonhoeffer, 2001; Rubin and Katz, 1999; Uchida and Mainen, 2003) and in the mouse (Abraham et al., 2004; Belluscio and Katz, 2001; Wachowiak and Cohen, 2003), an odour activates a combination of glomeruli (blue and green spots in Fig. 1C).

We also performed fluorescence recording in vivo (Fig. 1D) on a mouse model that genetically express the synaptopHfluorin $(\mathrm{SpH})$ protein in the sensory neurons synaptic terminals (Fig. 1E, Bozza et al., 2004). SpH corresponds to the fusion protein between the synaptic vesicle protein VAMP-2 and a green fluorescent protein $\mathrm{pH}$-sensitive (Miesenbock et al., 1998). Upon odour stimulation, vesicular release at the terminal induces an observable increase of fluorescence that reveals the pattern of nervous inputs to the OB (Fig. 1F, Bozza et al., 2004).

Both intrinsic and $\mathrm{SpH}$ recordings revealed glomerular activity, but up to now, little has been done to model and assess the statistical relevance of this activity. In addition, glomeruli identification is generally performed manually and can thus be subject to human bias. To solve these problems, we propose to build linear models of the signal for both recordings (i.e. to mathematically define the expected shape of the signals), and to use this a priori knowledge for statistical detection of glomeruli pattern.

\section{Building linear models (LMs) for optical imaging recordings}

In short, a linear model is a description of the signal by an ensemble of time dependant functions (regressors; see mathematical definition below), whose shape (time-course) is fixed, but whose amplitudes can vary to best fit the data. Obviously to get an appropriate model, the number of functions to include and their shape depend on the signal considered. In the absence of precise biophysical knowledge about the recorded signals (the mechanisms for the intrinsic signals are unclear; there is no quantitative 
measurements of olfactory nerve release in a freely breathing animal to use for $\mathrm{SpH}$ ), we decided to start with purely phenomenological models. In this section, we present the general strategy that we used to construct these models and to evaluate their precision.

\section{Mathematical background}

Given a set of (linearly-independent) regressors $\left\{f_{k}(t)\right\}$, the signal $s(t)$ can be explained as a sum $s(t)=\sum_{k} \alpha_{k} f_{k}(t)+r(t)$, where $r(t)$ is the residual. For discrete signals, the model can be reformulated as $\boldsymbol{s}=\mathbf{F} \boldsymbol{\alpha}+\boldsymbol{r}$, where $\boldsymbol{s}$ is the signal vector, $\boldsymbol{\alpha}$ the parameter vector, $\boldsymbol{r}$ the residual vector, and $\mathbf{F}$ the design matrix that contains the regressors as columns.

Under the assumption of this signal model with only white noise left in the residual, the best linear unbiased estimate of the coefficients $\alpha$ is given by the least-squares estimate: $\boldsymbol{\alpha}=\left(\mathbf{F}^{T} \mathbf{F}\right)^{-1} \mathbf{F}^{T} \boldsymbol{s}$ and its residual $\boldsymbol{r}=\mathbf{F} \boldsymbol{\alpha}-\boldsymbol{s}$.

The parameter of interest is extracted by using a contrast vector $\boldsymbol{c}$ as $u=\boldsymbol{c}^{T} \boldsymbol{\alpha}$ with noise variance estimate $\sigma_{u}{ }^{2}=\boldsymbol{r}^{T} \boldsymbol{r} \boldsymbol{c}^{T}\left(\mathbf{F}^{T} \mathbf{F}\right)^{-1} \boldsymbol{c}$. Standard statistical testing procedures can then evaluate the presence of this regressor.

\section{Methods for determination of the regressors}

The number of functions to include in a linear model should be as large as the number of different processes contained in the data, plus a constant function to describe resting levels. As an example, photobleaching and stimulus dependant fluorescence increase are two processes presents in fluorescence recordings. The idea is indeed to avoid incomplete models, which would lead to fitting errors because the white noise hypothesis for the unexplained part of the data would not be fulfilled. For the same reason, temporally correlated noise should also be minimized in the recordings.

For a phenomenological description only observation of the data can help in finding the different processes and the functions that would best describe their time-course. Then if these functions have non-linear parameters (e.g. time constant for an exponential), they can be determined by optimization on an independent pool of data. In this article, we used two strategies for optimization. First, if the process for which we wanted to find a regressor could be isolated from other processes (e.g. photobleaching can be isolated in the absence of stimulus), we performed non-linear fittings of the desired function using the Levenberg-Marquardt algorithm. Second, we also directly optimized the linear models by performing linear fittings for a large set of non-linear parameter values, and finding the combination that yields the minimum residual. In both cases, optimization was performed individually on each experiment of the reference pool and the variability of the optimal parameters was observed, which gave an indication on the expected error due to the choice of fixed non-linear parameters.

\section{Wavelet-based statistical analysis}

\section{Motivation for using the wavelet transform}

Once the LM has been set up, the next step is to evaluate the significance of the linear fittings (e.g. to determine whether the weight of the regressor associated to neural activity is only due to noise). One method could be to apply the LM and evaluate the significance for each pixel of the image. However, glomeruli signal is distributed over clusters of several pixels. Such spatial correlation is not exploited by a simple pixel-by-pixel approach, thus resulting in a statistical threshold too conservative. The idea of the wavelet transform is to decompose the signal with a set of functions (wavelet basis functions), which represent image features at different scales. Performing the LM fitting and the statistical test in the wavelet domain (i.e. after decomposition) allows for fitting the regressors' weights for these clusters, and therefore gives a larger sensitivity.

Another possible approach exploiting the spatial correlation in the data could be the application of a Gaussian smoothing filter that corresponds to the typical size of the glomeruli (i.e. matched filter argument). However, such an approach brings along several problems.

1. Smoothing removes potentially interesting spatial information.

2. The statistical interpretation becomes more complicated: while the apparent SNR increases, the statistical significance needs to take into account the correlation that has been introduced.

3. Tuning of the width of the Gaussian filter is non-trivial.

4. Strong stimuli often induce a global response of the bulb (in particular for the intrinsic signal). It is important to be able to distinguish local activation from background activation.

The multi-resolution decomposition naturally provided by the spatial wavelet transform seems to be well adapted for dealing with these shortcomings.

1. No information is lost when taking the transform.

2. The statistical interpretation is mapped into the spatial domain by the framework that we employ (Van De Ville et al., 2004).

3. The equivalent smoothing at each scale of the transform is adaptive and allows us to deal with the different "resolutions" of the spatial patterns present in the data.

4. Wavelets automatically capture the difference of the signal with respect to its local neighbourhood. In this case, the global background signal resides in the lowpass sub-band only.

The wavelet decomposition has been used before for the analysis of neuro-imaging data; in particular, for PET and fMRI (see Ruttimann et al., 1998; Turkheimer et al., 2000; Bullmore et al., 2003; Van De Ville et al., 2006 for an overview). An important advantage of the present framework (Van De Ville et al., 2004) is that it allows us to put the statistical test in the spatial domain.

\section{Spatial representation by the wavelet transform}

The discrete wavelet transform (DWT; Mallat, 1989, 1999) decomposes the signal into a weighted sum of basis functions. These are shifted and dilated versions of a (bandpass) wavelet: $\psi(x)$, and shifted versions of a (lowpass) scaling function $\varphi(x)$. The wavelet functions are capturing the details of the image with a given typical size which depends on the sub-band they are belonging to (the first sub-band contains the smallest wavelets, and then the size increases in the successive sub-bands). The scaling functions are capturing the details of the image which are larger than the largest wavelet function. For the ease of notation, we write the 2-D spatial wavelet decomposition of an image $v(\boldsymbol{n})$, as

$v(\boldsymbol{n})=\sum_{\boldsymbol{k}} v_{\mathrm{w}}(\boldsymbol{k}) \psi_{\boldsymbol{k}}(\boldsymbol{n})$

where $\boldsymbol{k}$ runs over all sub-bands and orientations, including the low-pass one, and $\psi_{\boldsymbol{k}}$ is the corresponding basis function (which account for both $\psi$ 's and $\varphi$ 's). The $v_{\mathrm{w}}(\boldsymbol{k})$ 's are the wavelet 
coefficients of the decomposition which contain all the information about the original image but in a scale and position dependant manner. Note that the wavelet transform is linear, therefore it is equivalent to apply the (temporal) linear model to the (spatial) wavelet decomposition.

\section{Implementation of the statistical framework}

The wavelet-based statistical analysis proceeds as follow (see Fig. 5):

1. Each frame in the image series is decomposed using the orthogonal cubic B-spline DWT (6 dyadic decomposition levels). We obtain a time-course for every wavelet coefficient.

2. The LM is fitted to the time-course of each wavelet coefficient, yielding estimates for the parameters and the noise variance. For each wavelet coefficient with index $\boldsymbol{k}$, the parameter of interest $u_{\mathrm{w}}(\boldsymbol{k})$ (e.g. amplitude of activation) and its noise standard deviation $\sigma_{\mathrm{w}}(\boldsymbol{k})$ are extracted. Then, the $t$-values defined as $t_{\mathrm{w}}(\boldsymbol{k})=u_{\mathrm{w}}(\boldsymbol{k}) / \sqrt{\sigma_{\mathrm{w}}^{2}(\boldsymbol{k}) / J}$, with $J$ representing the degrees of freedom, should follow a Student $t$-distribution in the absence of a response.

3. Adaptive denoising is performed based on the $t$-value of each wavelet coefficient. Specifically, coefficients with a $t$-value below a certain threshold $\tau_{\mathrm{w}}$ are set to zero: if $\left|t_{\mathrm{w}}(\boldsymbol{k})\right|<\tau_{\mathrm{w}}$, we put $\tilde{u}_{\mathrm{w}}(\boldsymbol{k})=0$ otherwise $\tilde{u}_{\mathrm{w}}(\boldsymbol{k})=u_{\mathrm{w}}(\boldsymbol{k})$.

4. The thresholded parameter map is reconstructed as $\tilde{u}(\boldsymbol{n})=\sum_{\mathbf{k}} \tilde{u}_{\mathrm{w}}(\boldsymbol{k}) \psi_{\boldsymbol{k}}(\boldsymbol{n})$ and the noise estimation as $\sigma(\boldsymbol{n})=\sum_{\boldsymbol{k}} \sigma_{\mathrm{w}}(\boldsymbol{k})\left|\psi_{\boldsymbol{k}}(\boldsymbol{n})\right|$.

5. The hypothesis test in the spatial domain relies on the following null hypothesis rejection inequality: $P\left[\frac{\tilde{u}(n)}{\sigma_{\mathrm{u}}(n)} \geq \tau_{\mathrm{s}}\right] \leq Y\left(\tau_{\mathrm{w}}, \tau_{\mathrm{s}},\right)$, where $Y\left(\tau_{\mathrm{w}}, \tau_{\mathrm{s}}\right)$ is data-independent. The threshold values $\tau_{\mathrm{w}}, \tau_{\mathrm{s}}$ are obtained by fixing $Y\left(\tau_{\mathrm{w}}, \tau_{\mathrm{s}}\right)=p / V$, where $p$ is the desired global significance level and $V$ the number of pixels (for more detail see Van De Ville et al., 2004). It is important to notice that this result is conservative; i.e. we did not make any assumption on the (remaining) correlation in the wavelet domain, which assures a strong control over the false positive fraction.

\section{Extension for multi-resolution analysis}

Since the wavelet decomposition is made up of wavelets with different dilations, it is able to capture patterns of different sizes. Each sub-band of the DWT groups together coefficients corresponding to wavelets with the same dilation. For the orthogonal B-spline DWT of degree $\alpha$ (we used $\alpha=3$ in this study: the typical basis functions are represented in Fig. 7A), it is possible to evaluate the characteristic size of the wavelets in each sub-band. Indeed, according to (Van De Ville et al., 2003) and (Fadili and Bullmore, 2004), we could use the following formula for the diameter $D_{\text {eq }}$ (measured at half-maximum in pixels) of the equivalent Gaussian in a low-pass sub-band after $J$ iterations:

$D_{\text {eq }}(J)=\sqrt{2 \ln 2} \sqrt{\alpha+1} \sqrt{\frac{4^{J}-1}{3}}$

For the 6 iterations of the DWT of this study and for a resolution of $12.5 \mu \mathrm{m}$ per pixel, we can define six low-pass sub-bands with $D_{\text {eq }}$ respectively equal to $30,66,135,271,544,1088 \mu \mathrm{m}$.

Interestingly by a simple extension of the above described wavelet-based statistical framework, we can obtain a statistical test for a reconstruction using a limited number of sub-bands, which restricts the detected patterns by an implicit a priori on the size and shape.

For this purpose, we should recognize that the rejection inequality is still valid when we replace $\tilde{u}(\boldsymbol{n})$ by $\min$ $\left(\sum_{\boldsymbol{k}} \tilde{u}_{\mathrm{w}}(\boldsymbol{k}) \psi_{\boldsymbol{k}}(\boldsymbol{n}), \sum_{\boldsymbol{k} \in S} \tilde{u}_{\mathrm{w}}(\boldsymbol{k}) \psi_{\boldsymbol{k}}(\boldsymbol{n})\right)$, where $S$ is a set of indices corresponding to a selection of wavelet coefficients (e.g. a given sub-band). It is easy to see that these maps are at least as conservative as the full reconstruction. This means that a combination, using the minimum operator, of the complete reconstruction with any partial reconstruction will still result in a conservative test.

The method exploring the multi-resolution structure proceeds as follows. We combine the complete reconstruction with partial reconstructions that successively exclude one more sub-band at a time (each "sub-band" includes all orientations). In particular, we start by removing the low-pass sub-band, then the next band-pass, and so on and so forth. By successively removing these subbands (and making the proposed conservative combination), we obtain maps that contain consistent details of the remaining bandpass sub-bands (i.e. with smaller and smaller maximal typical size).

This method is particularly interesting for olfactory bulb data because glomeruli are clearly restricted in size and shape (see Fig. 1).

The Matlab code of the complete method (linear model fitting and wavelet-based statistical detection), including a working example, is freely available at http://bigwww.epfl.ch/demo/ olfactif/.

\section{Glomeruli detection methods}

\section{Automated procedure}

For automated detection, we identified glomeruli as local hills (or local dips for the negative intrinsic signal; see Fig. 1) of the image with a diameter in the range of the observed size of glomeruli in mice $(93.5 \pm 22$; mean \pm S.D. taken from Bozza et al., 2004). To make sure that these hills (or dips) are significant details of the image, they were detected as the local extrema on the map of consistent details. Using the multi-resolution exploration of the statistical maps, we retained consistent details of the second bandpass sub-band. At this scale, the wavelets have an equivalent width of $D_{e q}=66 \mu \mathrm{m}$, which is close to the lower bound $(70 \mu \mathrm{m}$ : average minus 1 S.D.) that we expect for glomeruli.

Once relevant extrema were localized, we performed a shape analysis of the hills underlying them by an adapted fitting based on the raw parameter map. The purpose of the fit was to determine the visible extent of the hill and its shape characteristics. Two-dimensional quadratic functions were used for the fitting, as they naturally approximate the neighbourhood of an extremum. Taking a square of size L around the extremum, all possible quadratic forms (varying elongation by 1 pixel steps and orientation by $20^{\circ}$ steps) inside this square were linearly fitted. The square size $\mathrm{L}$ was also varied between 4 and 20 pixels (50 to $250 \mu \mathrm{m})$. Among this large pool of fits, we selected the one with the smallest relative residual error (ratio of the sums of squares of the residual and the quadratic form), which gave the best quadratic approximation of the hill. This simple method performed very well to determine the boundaries of the detected hills despite their variability in shape and size (see Fig. 1). It also allowed fitting the local hills independently of the surrounding ones. 
Alternative procedure (Gaussian band-pass filtering)

To compare our framework to a more simple method, we designed an alternative method using band pass filtered images (Gaussian filter between 12 and $125 \mu \mathrm{m}$ ) thresholded at integer multiples of its standard deviation ( $1 \sigma, 2 \sigma$ and $3 \sigma$ thresholds). Such method was in fact employed in some studies (Belluscio and Katz, 2001; Meister and Bonhoeffer, 2001) with a criterion of 2 S.D. These thresholded images yielded zones analogous to the above derived consistent details on which the detection step could be performed and then the shape fitting procedure (described above) was applied.

\section{Manual detection}

At last, the accuracy of our automated glomeruli detection method was evaluated with respect to manual selection performed by a human operator. We choose 10 presentations of different odorants or concentrations (repeated 4 times each) that visibly activated a large number of glomeruli, and asked independently three experienced users to extensively circle putative glomeruli on $\mathrm{SpH}$ activation images (using LM derived and divided images at the same time). The overlapping selections from all three detection sets were then used to build a consensus detection for each activation map. We also evaluated the deviation from this consensus (glomeruli detected by one user but not all three) as a measure of the inherent subjectivity of human selection.

\section{Results}

Determination and assessment of regressors for the linear models (LMs)

\section{Intrinsic signal}

Intrinsic activation signal obtained after standard data processing (see Methods, Fig. 2A) was characterized by a monotonous decrease in reflected light intensity during the odour application, and a slow recovery afterwards (Figs. 2B and C). In many cases, the decrease was particularly well fitted by a decaying exponential (Fig. 2C), and this for most region of the image. This time-course was consistent with previous observation of olfactory bulb intrinsic signal (Meister and Bonhoeffer, 2001; Spors and Grinvald, 2002; Wachowiak and Cohen, 2003), although in some studies clear exponential behaviour was seen only after high-pass spatial filtering. In our case and for stable recordings, no filtering was required to observe consistently this time-course. Moreover, no other consistent time-course was observed in the data. Thus an

\section{A $\quad$ Amylacetate $10 \%$}
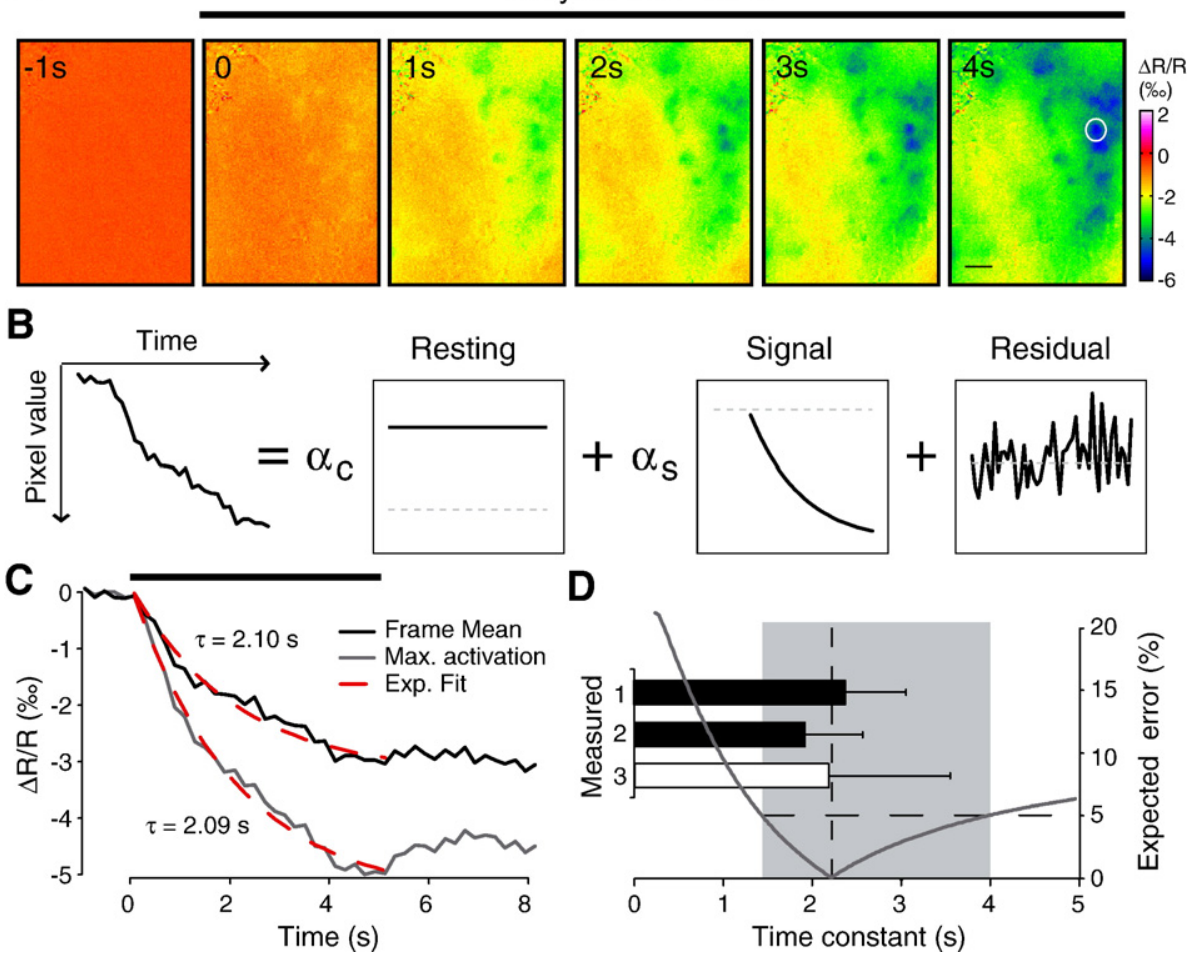

\section{D}

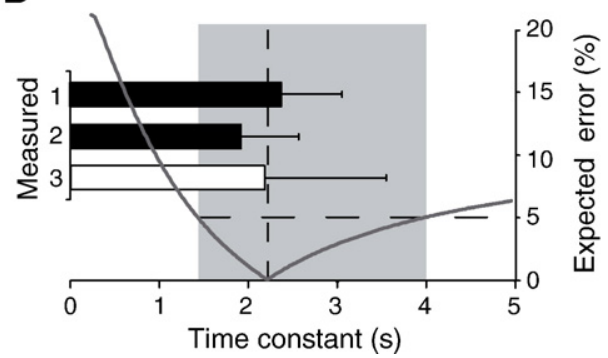

Fig. 2. Linear model for intrinsic imaging. (A) Time series of an intrinsic signal recording averaged over $1 \mathrm{~s}$ periods (obtained with the standard method: division by first frame). Amyl acetate $(10 \%)$ is presented when acquisition of the second frame begins. Scale bar $200 \mu$ m. (B) Schematic description of the linear model (LM) for intrinsic signal. On the left a typical time-course of the signal is shown. It could be decomposed into the sum of a constant component, an exponential decay and a residual. (C) Time-courses for the activated glomerulus (white circle) and for the frame average shown in (A). A non-linear exponential fitting is showed in superposition (dashed red lines). Fitted decay time constants are $2.10 \mathrm{~s}$ and $2.09 \mathrm{~s}$ for glomerulus and frame average respectively. (D) Histogram: Average decay time constants derived by fitting of the frame average (1) and frame maximum (2) time-courses. (3) is the value obtained by optimization of the linear model ( $n=510$ presentations; 10 animals). Curve: Error resulting from the linear fitting of a decaying exponential with a time constant of $2 \mathrm{~s}$ on an exponential with a different time constant ( $x$-axis). The grey shading represents the time constant range for which error is below $5 \%$ of signal amplitude. 
adequate model for intrinsic signal can be simply composed of a constant component and a decaying exponential (see also schematic Fig. 2B):

$s(t)=\alpha_{\mathrm{c}}+\alpha_{\mathrm{s}}\left(1-e^{\left(t-t_{0}\right) / \tau}\right) / \theta\left(t-t_{0}\right)$,

where $t_{0}$ is the stimulus onset time and $\theta$ is the step function. For the model to be linear, one needs to fix the value of the time constant $\tau$. To evaluate $\tau$, we performed non-linear fittings of the activation time-course for the maximally active region (e.g. Fig. 2A selected region) and for the mean of the frame. On a large data set composed of various odorant presentation at different concentration (10 animals; $n=510$ presentations), the average fitted time constant was $1.91 \pm 0.71 \mathrm{~s}$ (mean \pm S.D.) for the strongest activation and $2.35 \pm 0.74 \mathrm{~s}$ for the frame mean (Fig. 2D). When the images were spatially high-pass filtered (cut-off frequency at $1 / 100$ ), we observed a decay time of $1.70 \pm 0.50 \mathrm{~s}$ for the maximum. Finally, to confirm our estimation, we made LM fittings on the same dataset with systematic variation of $\tau$. On average, the time constant that yielded the lowest residual was $2.18 \pm 1.36 \mathrm{~s}$.

Following the latter value, we decided to set $\tau=2.2 \mathrm{~s}$. However, the fitting analysis has revealed some substantial variability, which might be due to external perturbations of the signal (noise) but also to intrinsic time constant jitters. What is then the error expected from the LM fitting if the time constant is actually different? To answer this question we fitted our linear model to decaying exponentials function with time constants comprised between 0 and $5 \mathrm{~s}$. The error was found to be below $5 \%$ of signal amplitude for decays between $1.4 \mathrm{~s}$ and $4 \mathrm{~s}$ (Fig. 2D). Therefore comparing this interval with the observed variability of the signals, we can be confident that time constant heterogeneities will have little impact on LM analysis.

\section{SpH signal}

Compared to intrinsic signal, $\mathrm{SpH}$ fluorescence (Fig. 3A) had a rather complex behaviour. A typical raw time-course showed a consistent decay (due to photobleaching) followed by a rise upon odour stimulation (see Fig. 3B). However, when the photobleaching was corrected by standard methods the observed signal was still complicated. Glomerular activation clearly induced a fluorescence increase over time that lasted after odour stimulation as previously reported (Fig. 3A, Bozza et al., 2004). But in general, a transient dip locked to odour onset preceded the rise in fluorescence (see Fig. 3D, red trace). In some recordings, the transient dip and the rise in fluorescence could be seen almost independently at different locations of the $\mathrm{OB}$ (see selected zones
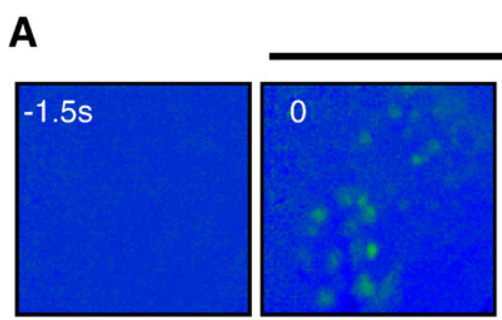

Ethylbutyrate $50 \%$
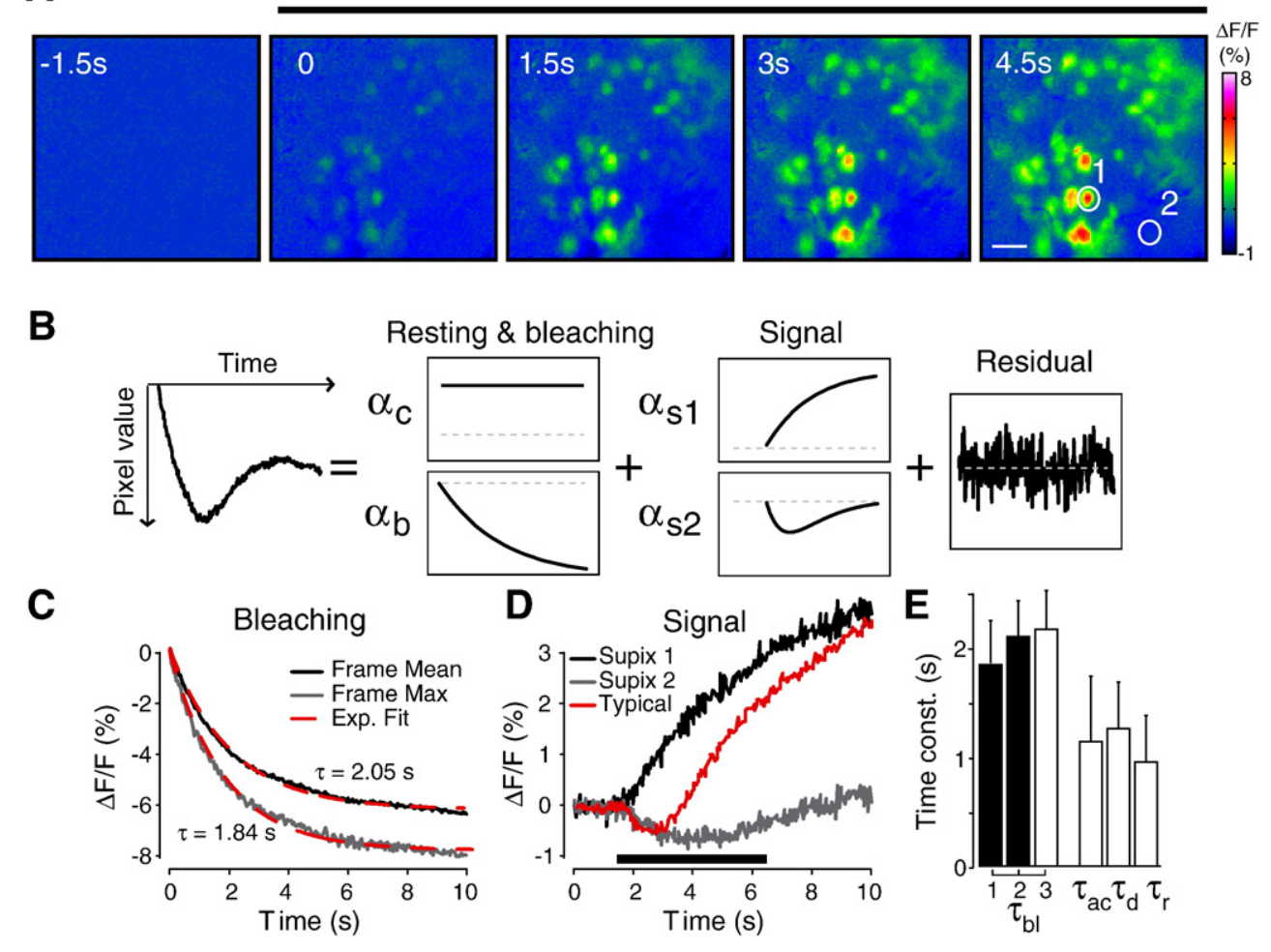

Fig. 3. Linear model for $\mathrm{SpH}$ signals. (A) Time series of a SpH recording obtained after subtraction of a "blank" series (standard method) and averaged over $1.5 \mathrm{~s}$ periods. Ethyl butyrate (50\%) is presented at the beginning of the second frame. Scale bar $200 \mu \mathrm{m}$. (B) Sketch of the linear model for SpH signal. The typical time-course showed on the left is decomposed into four components and a residual accounting for resting fluorescence, bleaching, odour-induced fluorescence rise and transient activation respectively. (C) Typical time-course of photobleaching for the zone of maximal resting fluorescence (grey) and frame average (black) of the bulb shown in (A). Corresponding exponential fittings are showed in red dashed lines. (D) Time-course of odour evoked signal for the two regions of interest shown in (A) (region 1: black, region 2: grey). These time-courses were in fact rather unusual in our dataset. A more typical activation time-course is showed in red. (E) Summary histogram of the LM time constants. Bleaching time constant $\left(\tau_{\mathrm{bl}}\right)$ measured on the time-course of the most fluorescent zone (1) and of the frame average (2). (3) is obtained by optimization of the linear model on blank presentations ( $n=766$ trials; 5 animals). The activation time constant $\tau_{\text {ac, }}$, and the two time constants of the transient component (rise: $\tau_{\mathrm{r}}$ and decay: $\tau_{\mathrm{d}}$ ) are obtained by optimization. 
Fig. 3A and their time-courses Fig. 3D), suggesting a superposition of two different phenomena. While the rise in fluorescence is caused by vesicular release at olfactory nerve terminals, we do not have yet an explanation for the transient dip. Several hypotheses could be proposed, going from activity dependant changes in fluorescent light absorption by the tissue (intrinsic signal), to transient changes in extracellular acidity due to release as observed in slice experiments (McGann et al., 2005; with the restriction that the latter phenomenon seems faster and should be more spatially restricted than the transient dip). Nevertheless it was clear that a linear model of $\mathrm{SpH}$ signal should have four components: resting fluorescence level, photobleaching, odour induced fluorescence rise and transient dip (Fig. 3B).

Similarly to the intrinsic signal, photobleaching had a decaying exponential time-course that could be clearly revealed when no stimulus was presented (Fig. 3C). Non-linear fitting over a large data set ( 5 animals; $n=766$ blank recordings) gave average time constant values of $1.86 \pm 0.32 \mathrm{~s}$ for the most fluorescent zone $(5 \times 5$ pixels zone around maximum) and $2.11 \pm 0.38 \mathrm{~s}$ for the frame average (Fig. 3E). We also made LM fittings of the bleaching signal with systematic variations of the decay time. Consistently, the average "best" time constant was $2.18 \pm 0.35 \mathrm{~s}$ (Fig. 3E). Therefore, we finally set $\tau_{\mathrm{bl}}=2.2 \mathrm{~s}$ for the bleaching time constant. It is noteworthy that, unlike for intrinsic signal activation, the variability of fitted $\tau_{\mathrm{b}}$ had some identifiable structure. Aside from fluctuations, we observed a clear correlation between resting fluorescence levels and bleaching decay time. Spatially, the zone with higher resting fluorescence had faster decay. Moreover, repeated illumination of the preparation along several hours, decreased the average fluorescence level which in turn increased the average decay time. Although this resulted in an observable difference, it never yielded time constants after fitting lower than $1.5 \mathrm{~s}$ or higher than $2.8 \mathrm{~s}$. Thus, according to our error analysis reported before, these effects do not account for more than $5 \%$ fitting error.

For the two remaining components, it was clearly hazardous to try to fit directly their time-course on selected zones, because they mostly appear in superposition. Even when they seemed to be rather separated, the transient dip could still be hidden in the activation and slow down its dynamic (as might happen in Fig. 3D). Observations and preliminary fittings indicated that activation could be modelled by a simple exponential rise (time constant $\tau_{\mathrm{ac}}$ ), and that the transient dip had the shape of the difference of two exponentials (time constants rise $\tau_{\mathrm{r}}$ and decay $\tau_{\mathrm{d}}$ ). Thus the proposed LM for $\mathrm{SpH}$ signal reads:

$$
\begin{aligned}
s(t)= & \alpha_{\mathrm{c}}+\alpha_{\mathrm{b}}\left(1-\mathrm{e}^{-t / \tau_{\mathrm{b}}}\right)+\alpha_{\mathrm{s} 1}\left(1-\mathrm{e}^{-\left(t-t_{0}\right) / \tau_{\mathrm{ac}}}\right) \theta\left(t-t_{0}\right) \\
& +\alpha_{\mathrm{s} 2}\left(\mathrm{e}^{-\left(t-t_{0}\right) / \tau_{\mathrm{d}}}-\mathrm{e}^{-\left(t-t_{0}\right) / \tau_{\mathrm{r}}}\right) \theta\left(t-t_{0}\right)
\end{aligned}
$$

where the time constants $\tau_{\mathrm{ac}}, \tau_{\mathrm{r}}$ and $\tau_{\mathrm{d}}$ have to be determined. To do so, we selected a pool of $n=100$ robust responses ( 5 animals) and performed fittings of the latter LM with systematic variation of the three remaining parameters. Values optimized by this method were $\tau_{\mathrm{ac}}=1.15 \pm 0.6 \mathrm{~s}, \tau_{\mathrm{r}}=0.96 \pm 0.43 \mathrm{~s}$ and $\tau_{\mathrm{d}}=1.26 \pm 0.43 \mathrm{~s}$ and were retained for all subsequent $\mathrm{LM}$ analysis (Fig. 3E).

However, the choice of optimal time constants does not ensure that the LM is globally valid and accurate. To be sure that the fitting of our LM was appropriate to extract odour induced signals, we had to evaluate how precise LM-based reconstructions were in comparison to signal variability.

\section{LM-based signal extraction for SpH fluorescent signal}

The purpose of "blank" subtraction (or division) is to remove non-specific signal components and reveal stimulus-triggered
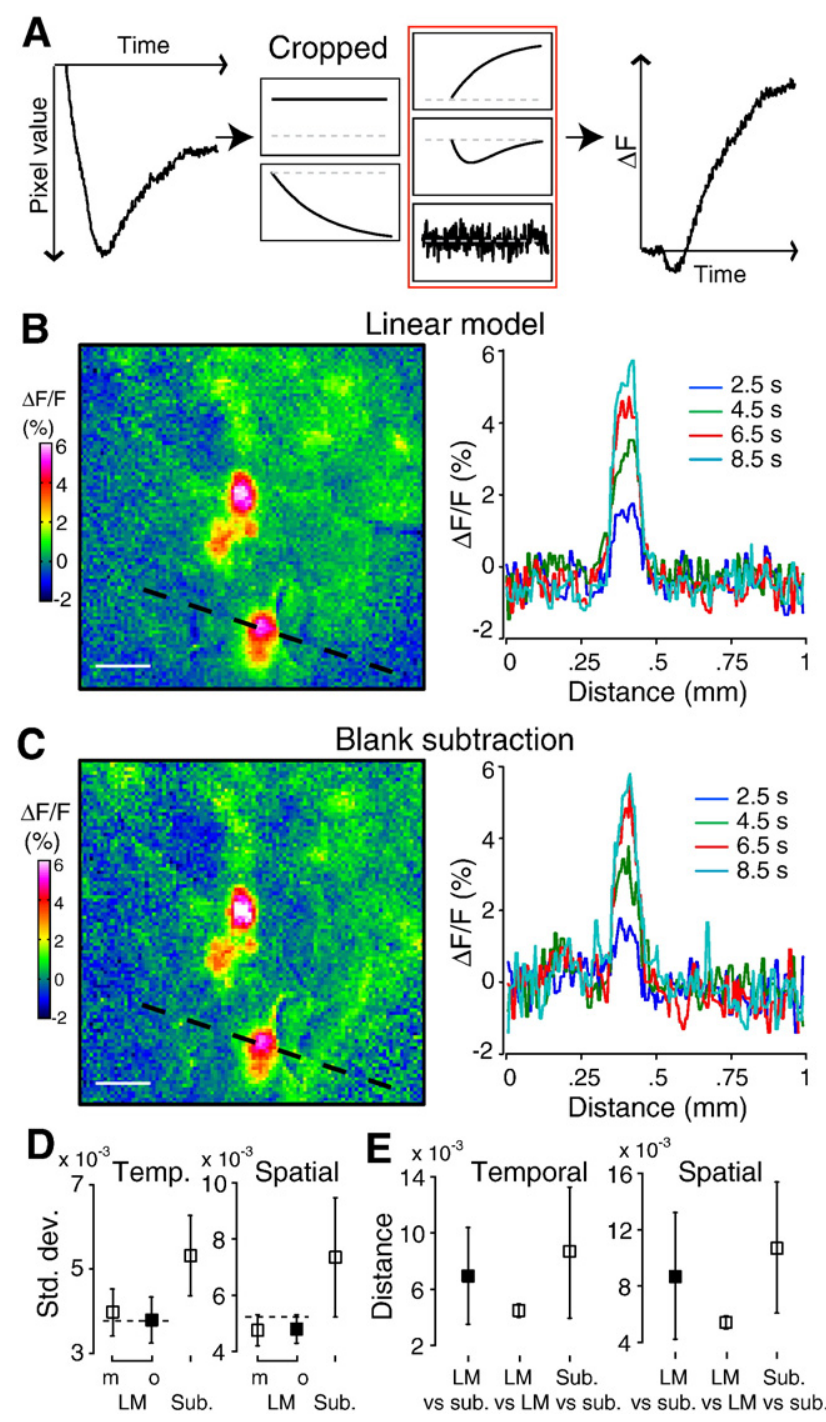

Fig. 4. Reduced noise in the linear model reconstructed images. (A) Extraction of the signal of interest without blank subtraction for $\mathrm{SpH}$ signal. After fitting the LM, constant and bleaching components are discarded and the time series is reconstructed. (B) Left: Single frame ( $5 \mathrm{~s}$ after stimulus) of a SpH recording (amyl acetate $2 \%$ ) obtained by LM fitting and reconstruction. Right: Spatial amplitude profile along the black dashed line drawn on the left. Scale bar $200 \mu \mathrm{m}$. (C) Same as B but for the standard image treatment method (subtraction of a blank recording). (D) Average spatial and temporal standard deviations for a dataset of $n=766$ blank $\mathrm{SpH}$ recordings in 5 animals. LM based photobleaching correction (LM: m,o) is compared to the subtraction method (Sub; subtraction of two consecutive recordings). The value (o) is obtained by taking for each recording the decay time constant that minimize temporal residual, whereas $(\mathrm{m})$ is obtained with the average time constant. (E) Average Euclidean distance (normalized by vector size) between repetitions of the same stimulus ( $n=150$ comparisons in 5 animals) evaluated in time and space. LM treatment is compared to the blank subtraction method (LM vs. LM compared to Sub vs. Sub). In addition, distance between LM and subtraction method is measured $(n=100$ comparisons in 5 animals). All discrepancies between LM treatment and blank subtraction in (D) and (E) are statistically significant ( $t$-test, $p<0.001)$. 
signals of interest. Since the LM captures separately specific and non-specific components, they can also be used to replace the standard blank subtraction procedure. After fitting the complete LM, one can simply discard non-specific regressors (i.e. the constant component for intrinsic, and the constant and the bleaching components for $\mathrm{SpH}$ fluorescence) and reconstruct the time series with the remaining ones and the residual (see schematic Fig. 4A). This procedure relies on the accuracy of the discarded non-specific regressors. We observed that indeed this is the case for both the constant component (intrinsic and $\mathrm{SpH}$ ) and the bleaching component ( $\mathrm{SpH}$ only). Moreover, it is easy to understand that the LM-based signal extraction results in less noisy time-courses compared to "blank" subtraction, because they do not contain the additional variability of the "blank" signal itself. Theoretically, the prediction translates into a $\sqrt{2}$ reduction of noise standard deviation (S.D.). Moreover, if the noise is independent from pixel to pixel, diminution of temporal fluctuations also reduces spatial noise.

Visual inspection of the LM-based signal extraction for $\mathrm{SpH}$ recordings shows a diminution of pixel noisiness (Fig. 4B vs. C). We then quantified noise S.D. by computing the S.D. of blank image series $(n=766$ blanks, in 5 animals). For the standard treatment (subtraction of two successive blanks), temporal and spatial S.D.s (expressed in \%o) were $5.3 \pm 0.9$ and $7.3 \pm 2$ respectively (Fig. 4D), whereas for the LM treatment (linear fitting of photobleaching), they were close to the predicted $\sqrt{2}$ reduction (time: $4 \pm 0.6$; space: $4.7 \pm 0.5$; Fig. 4D). Interestingly, if the optimal bleaching time constant for each trial was used instead of the fixed value, the S.D. was not improved (time: $3.8 \pm 0.5$; space: $4.8 \pm 0.5$; Fig. 4D). At last, we evaluated the variability of odour induced response. We selected 25 odour responses (among 5 different animals) which were repeated 4 times each. We then computed the Euclidean distance (also expressed in \%o) between each repetitions (6 pairs for each odour response, so $n=150$ comparisons in total) after signal treatment. Again LM derived images were less variable than their "blank" subtracted counterpart (LM, time: $4.4 \pm 0.3$ space: $5.7 \pm 0.4$; subtracted, time: $8.6 \pm 3.5$ space: $11 \pm 4.5$; Fig. 4E). Finally, for the same presentation, distance between the LM derived and subtracted images is comprised between LM and subtracted images variability (time: $6.9 \pm 3.4$ space: $8.9 \pm 4.4$; Fig. 4E). Therefore, signal extraction using the full LM was consistent from trial to trial and did not induce fitting errors larger than the otherwise observed signal variability.

In conclusion, the $\mathrm{SpH}$ signal model appears to be suitable for explaining the observed data. Importantly, when used to extract relevant activations out of the raw signal, it yielded as expected a substantial reduction of noise as compared to standard methods. In addition, our analysis demonstrated that the chosen LM was a relevant description of the data. Therefore we could use it for further interpretation of the recorded signals.

\section{Wavelet-based statistical analysis}

Given the predictive power of our LMs, it is meaningful to employ them for statistical analysis of odour activation maps. The assessment could be performed pixel-by-pixel but such a simple approach would neglect spatial correlations in the signal and would be too conservative. Therefore, we used a statistical framework that combines the LM fitting with a spatial wavelet decomposition (see Methods and Fig. 5). To summarize, the main idea of this framework is to select wavelet coefficients (i.e. groups of pixels that correlate well with the wavelet) which follow the typical activation time-course by setting a threshold on their $t$-value (amplitude divided by S.D.) after LM fitting (Fig. 5, denoising step: the main point being that smaller wavelet coefficients capture mainly pixel noise and are rejected). Subsequently, the image is reconstructed and the framework gives a second threshold to reject pixels that are not significantly activated (Fig. 5, statistical test).

\section{Output of the wavelet-based statistical framework}

We applied our statistical framework to typical intrinsic signal and $\mathrm{SpH}$ recordings. As a result, we obtain maps of statistical significance for the parameter of interest, which were thresholded at a desired confidence level $p$. The confidence level was fixed at $0.1 \%$ with Bonferroni correction; i.e. the probability of finding one false positive pixel for the complete image is only of $1 / 1000$.

For both intrinsic and $\mathrm{SpH}$ signals, significantly active zones (significant amplitude of the stimulus triggered exponential decay or rise for intrinsic and $\mathrm{SpH}$ respectively) very often spanned the entire dorsal part of the bulb (Figs. $6 \mathrm{~A}_{2}, \mathrm{C}_{2}, \mathrm{D}_{2}$ ). This was not surprising for the intrinsic signal which is known to have a strong

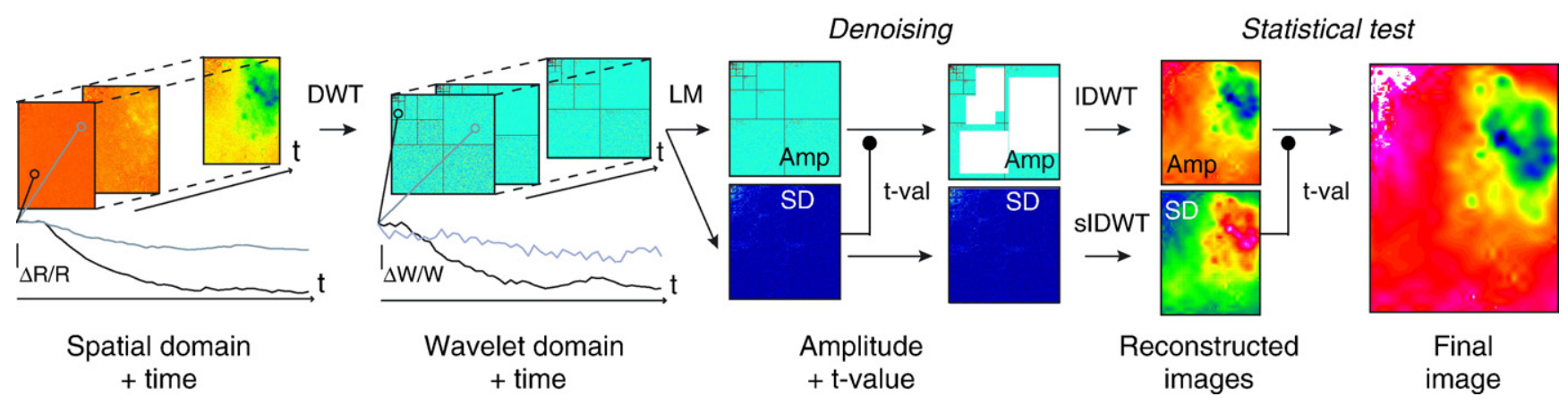

Fig. 5. Sketch of the wavelet based statistical framework for our recordings. From left to right: the time lapsed image series is spatially decomposed by a discrete wavelet transform (DWT). The linear model (LM) of the signal is then applied on the series of wavelet coefficients, resulting for each wavelet coefficient in an estimate for the parameter and the noise standard deviation (S.D.). Coefficients with a $t$-value (amplitude divided by S.D.) below some threshold are discarded (adaptive denoising); the image is then reconstructed by the inverse wavelet transform (IDWT). Spatial S.D. is derived by a special inverse transform (sIDWT). Finally, a significance threshold on the $t$-values in the spatial domain removes non-significant pixels that are represented in white (zero amplitude) in this example (for more details see Methods). 
SynaptopHfluorine

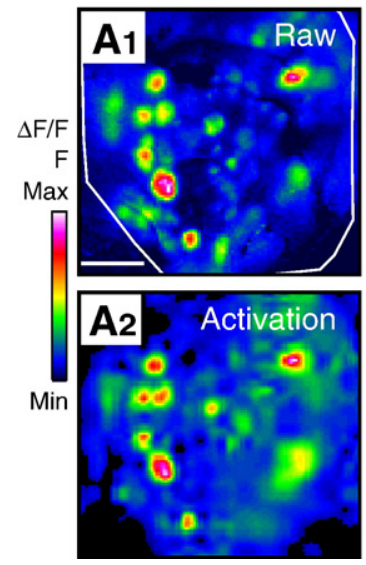

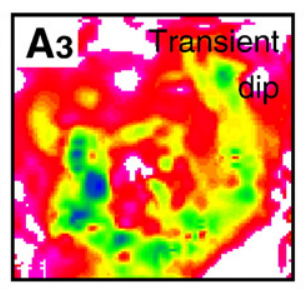

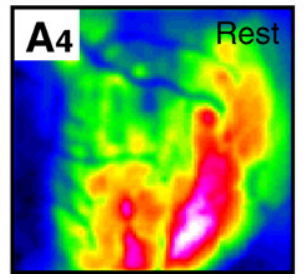

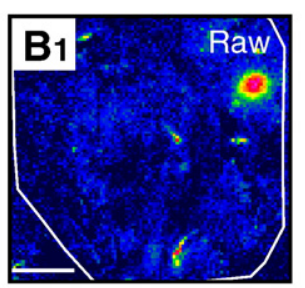

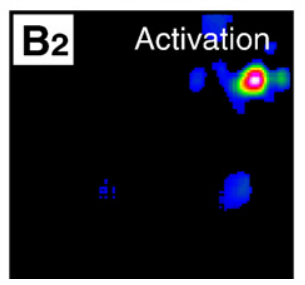

Intrinsic
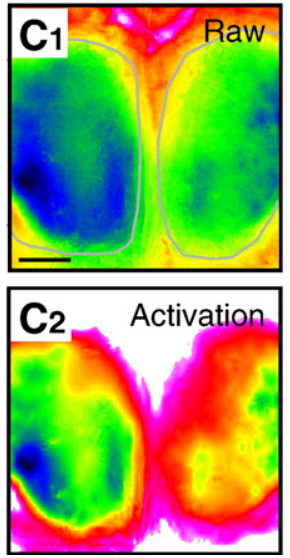

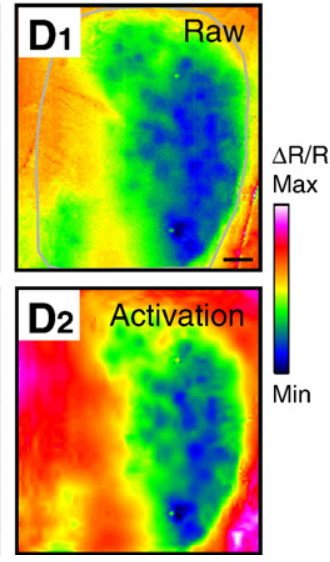

Fig. 6. Decomposition and statistical filtering of odour maps. (A) Example of statistically filtered and thresholded SpH data (single recording) compared to raw data. Methyl benzoate $(20 \%)\left(\mathrm{A}_{1}\right)$ Time average $(5 \mathrm{~s})$ of the raw data (with blank subtraction). Colour bar: 0 to $4 \%$. Scale bar $200 \mu \mathrm{m}$. ( $\left.\mathrm{A}_{2}\right) \mathrm{Map}$ of significant activations (exponential rise). Non-significant zones appear in black (i.e. zero amplitude). Colour bar: 0 to $6 \%$. ( $\mathrm{A}_{3}$ ) Map of significant amplitudes for the transient component of the signal. Non-significant zones appear here in white (i.e. zero amplitude). Colour bar: $-2 \%$ to $0 \%$. ( $A_{4}$ ) Extracted resting fluorescence pattern. Colour bar: 750 to 2000 (linear arbitrary units). $\left(B_{1}\right.$ and $\left.B_{2}\right)$ Same as $\left(A_{1}\right)$ and $\left(A_{2}\right)$ but for a lower concentration of methyl benzoate $(1 \%)$. Note that the extent of significant zone is much smaller than in (A). Colour bar: 0 to 3\%. (C,D) Examples of statistically filtered and thresholded intrinsic signal data (single recordings), compared to raw images. $\left(\mathrm{C}_{1}\right)$ Raw image ( $5 \mathrm{~s}$ time average) for simultaneous recording of left and right olfactory bulbs (OB). Odorant: amyl acetate $50 \%$. Colour bar: $-12 \%$ to $8 \%$. Scale bar $500 \mu \mathrm{m}$. $\left(\mathrm{C}_{2}\right)$ Corresponding statistical activation map. Non significant zones appear in white. Colour bar: $-12 \%$ to 0\%. $\left(\mathrm{D}_{1}\right)$ Raw map at higher resolution (left OB only; 5 s time average). Odorant: Hexanone $10 \%$. Colour bar: $-12 \%$ to $2 \%$. Scale bar $200 \mu$ m. $\left(\mathrm{D}_{2}\right)$ Corresponding statistical activation image. Contours in white or grey delineate the dorsal part of the olfactory bulb. Colour bar: $-12 \%$ to $-1 \%$.

diffuse component (Meister and Bonhoeffer, 2001). For the $\mathrm{SpH}$ signal, the large spread of significant regions is imputable to the low level of noise. As reported before (Bozza et al., 2004), a substantial amount of the fluorescence is scattered in the tissue or is out of focus. The effect of this light dispersion is a small diffuse signal that is significantly above noise. Indeed, if only few glomeruli are active the size of the significant zone strongly decreases (Fig. 6 $\mathrm{B}_{2}$ ). Maps of the transient component (fluorescence dip; example Fig. $6 \mathrm{~A}_{3}$ ) were also derived. They revealed a much more diffuse spatial pattern than the activation component (exponential rise). Interestingly, there was some correlation between this component and the resting fluorescence level. In general, the strongest dips were seen where basal fluorescence was brighter (compare Figs. $6 \mathrm{~A}_{3}$ and $\mathrm{A}_{4}$ ). Finally and as expected, the denoising step efficiently smoothed the activation maps, but induced little modifications of the spatial details (Fig. $6 \mathrm{~A}_{1}$ vs. $\mathrm{A}_{2}$, $B_{1}$ vs. $B_{2}, C_{1}$ vs. $C_{2}, D_{1}$ vs. $D_{2}$ ).

\section{Multi-resolution analysis of the statistical maps}

The framework also allowed us to explore the multi-resolution structure of the activation patterns. As described in the method, we can obtain a statistical test for a reconstruction using a limited number of wavelet sub-bands, which restricts the detected patterns by an implicit a priori on the size and shape. Starting from an example of full reconstruction of the $\mathrm{SpH}$ signal (Fig. 7B), we represented in Fig. 7C six selections of sub-bands with typical maximal size (largest equivalent width $D_{\text {eq }}$ ) respectively equal to $30,66,135,271,544,1088 \mu \mathrm{m}$ (see sketches of the wavelet space Fig. 7C: the larger typical size corresponds to the top left subband). As expected, when we removed low-pass sub-bands (as proposed for the sub-band selection algorithm), the significant zones in the maps of consistent details were more and more restricted to glomeruli, until the typical length became too small
$(30 \mu \mathrm{m})$, in which case almost no pixel was significant (Fig. 7C from top to bottom).

Using this method, we also measured the statistically significant multi-resolution content of $\mathrm{SpH}$ and intrinsic signal obtained from ethyl butyrate $(50 \%)$ and 3 -hexanone $(50 \%$ and $1 \%$ ) presentations $(n=3$ animals, 4 repetitions for each odour). For the complete decomposition, the size of the significantly activated zone was similar for both techniques and close to the complete frame that enclosed only two third of the OB (Figs. 7D-F). Significant $\mathrm{SpH}$ signal had a tendency to be more restricted than intrinsic, but the difference was rather small ( $20 \%$ of the complete frame at most). When removing successive low-pass and band-pass sub-bands, the significant area decreased monotonously for both techniques, showing that statistical analysis of the recordings was relying on the entire spatial frequency range.

However, $\mathrm{SpH}$ and intrinsic recordings revealed a different subband repartition of the signal. Measuring the amplitude of the maximally activated zone (i.e. most activated glomeruli), we observed that approximately $80 \%$ of its amplitude was due to the lowest band-pass sub-band (i.e. broader wavelets) for intrinsic, while it was only around $30 \%$ for $\mathrm{SpH}$ signal (Figs. 7D-F). The discrepancy was maintained when sub-bands corresponding to higher frequencies (and so narrower wavelets) were successively removed. This result indicates that a larger part of intrinsic signal originates from its diffuse component (consistently with Meister and Bonhoeffer, 2001), whereas $\mathrm{SpH}$ signal is more concentrated on the scales representative for glomeruli.

\section{Automated glomeruli detection}

As described in the Methods, the automated detection was performed in two steps. First we detected putative glomeruli on the map of consistent details (Figs. $8 \mathrm{~A}_{2}$ and $\mathrm{B}_{2}$ ) choosing a maximum equivalent width of $D_{\text {eq }}=66 \mu \mathrm{m}$ to have a good assessment of 
significantly distinguishable glomeruli (with $D_{\text {eq }}=135 \mu \mathrm{m}$, the next sub-band was not fine enough to assess specifically small peak-to-peak distances). Indeed, the results in Figs. 7C and 8A2 showed a very good separation of the extrema using the second sub-band, but not with the third. Second we tried to validate the detection and isolate the glomeruli with a shape fitting (see Methods and Figs. $8 \mathrm{~A}_{3}$ and $\mathrm{B}_{3}$ ).

\section{Validation of the detection for SpH signal}

The performance of our automated glomeruli detection method was evaluated with respect to manual selection performed by three human operators and to an alternative and simpler method based on Gaussian band-pass filtering and arbitrary thresholding (see Methods). 10 presentations of different odorants or concentrations (repeated 4 times each) were used for as tests recordings, and glomeruli detected by all three human operators were taken as a reference (consensus). Deviation from the consensus for human selection was on average as high as $35 \%$ of the number of consensus glomeruli (meaning around 4.5 non-consensus detections on an average of 13 consensus per odour). All three users showed some deviation even if one of them was a little more restrictive in his choice $(21 \%$ non-consensus detections against $45 \%$ and 39\%). Without any other treatment, the statistical detection (extrema in map of consistent details) captured $100 \%$ of the consensus glomeruli but also detected almost $80 \%$ additional glomeruli (Fig. 8D, first points), largely above human discrepancies. At the same time, the Gaussian filtering method could only capture a fraction of the consensus, and the best performing (i.e. threshold at 1 S.D.) yielded a very high error rate (more than $150 \%$ of consensus; Fig. 8D). We then used information provided by the shape fitting around the detected local maximum. In particular, we took as a parameter the signal to noise ratio (SNR) of the optimal quadratic function, which indicates if the detection is close to a bell shape form (as expected for glomeruli activation). We therefore put a threshold on the SNR and examined the remaining detections. For our statistical detection, the SNR threshold could be as high as 4 without affecting much the match with the consensus (97\%; Fig. $8 \mathrm{D}$, left). However, the deviation from consensus (sum of missed and added detections) dropped to $40 \%$, being then comparable to human detection. In comparison, the Gaussian filtering methods could not be rescued by the latter post-processing because error rates were too high (see Fig. 8D).

Fig. $8 \mathrm{C}$ shows a typical example of glomeruli detection on $\mathrm{SpH}$ signal. In this case, the wavelet based statistical method with postprocessing (threshold at 4) yielded a perfect match with the consensus. The Gaussian filtering method with a cut over 1 standard deviation found more glomeruli. Two of them (dashed dotted line) were clearly erroneous. The two others (dashed line) were in fact selected by one human operator and are typical non- consensus glomeruli. However, they were not even found in the map of consistent details, meaning that their relevance is arguable. Lastly and as reported elsewhere (Wachowiak and Cohen, 2001), many detections were missed for the Gaussian-filtering method with a threshold at 2 S.D.s.

The conclusion that we want to draw about extensive glomeruli detection can be well summarized by the histogram in Fig. 8E. Our multi-resolution statistical method detects as many glomeruli as human operators (around 17 on average). However, for humans about one-third of the detections are debated (consensus around 13 detections on average). Simpler methods solely based on spatial properties of the image are inappropriate (too many or too little detections). Altogether, these results show the usefulness of the statistical analysis for robust identification of activated glomeruli. They also suggest that human based mapping of odour activated glomeruli could be advantageously replaced by our fully automated method for improved speed and objectivity.

\section{Discussion}

We have introduced a multi-resolution wavelet analysis allowing us to make a statistical assessment of activation patterns of different sizes. This framework was particularly useful to detecting glomeruli-linked activations within a global response of the olfactory bulb. The statistical analysis was based on phenomenological temporal models of olfactory bulb optical imaging signals, which turned out to be a good prediction of the data. Such parametric methods are already widely used in other contexts (in particular, other brain imaging methods such as fMRI or PET), but have not yet been applied to optical imaging of animal brain to the best of our knowledge. Here, we also demonstrated the usefulness of the parametric approach for extraction of the intrinsic and synaptopHfluorin signals. In this section, we discuss the advantages and restrictions of the presented methods while evoking some extensions of the LM and the multi-resolution statistics to other optical imaging techniques. Then, focusing on the outcome for olfactory bulb physiology, we discuss the relevance of glomeruli detection based on our statistical framework. At the end, we briefly compare the performances of synaptopHfluorin versus intrinsic imaging techniques for observation of glomeruli activations.

\section{LM-based signal extraction: restrictions and applicability to other optical imaging techniques}

Signal extraction by standard methods for $\mathrm{SpH}$ and intrinsic imaging in the olfactory bulb could be replaced by LM fitting followed by reconstruction of the components of interest. Once the appropriate LM is determined, the latter method does not depend

Fig. 7. Multi-resolution analysis of the odours maps. (A) 1-dimensional profile of the wavelet and scaling functions that serve for the orthogonal cubic B-spline discrete wavelet transform (DWT). (B) Left, Map of the wavelet coefficients for odour activation derived from a SpH recording. Right. Corresponding filtered and thresholded activation map. Odorant: methyl benzoate 20\%. Colour bar: 0 to $5 \%$. Scale bar: $200 \mu \mathrm{m}$. (C) Assessment of activation details for the map in B. Successive high pass filtering steps are performed by removing one after the other the last iterations of the DWT (iterations are numbered from $J=1$ to 6 ). The grey square in the schematics (left) represents the coefficients that were discarded for filtering. They correspond to the largest scales of the image (low pass). After filtering, images can be reconstructed and the statistical threshold applied (images on the right; higher low cut from top to bottom; non-significant pixels are in black). For consistency, in a given step we only keep the zones that were also significant in the previous filtering steps. (D) Top. Size of the significant zone (expressed as fraction of the full image) for the successive filtering and assessments steps (each point correspond to the removal of one DWT iteration; first point: all sub-bands retained). Bottom. Amplitudes of the maximal significant activation for the successive filtering steps. Values are normalized by the first point (all sub-bands retained). Average and S.D. over $12 \mathrm{SpH}$ and intrinsic signal recordings (3 animals, 4 repetitions each). Presented odorant: ethyl butyrate $50 \%$. (E) Same as D but for hexanone $50 \%$. (F) Same as D but for hexanone $1 \%$. 
on the acquisition of reference images and it is more robust to noise. Even if our application here is rather specific, the LM fitting method is general and could be applied to many types of optical signals. Because it requires preliminary experiments to construct the model, our method cannot be used without any prior knowledge on the expected signal, but is more appropriate for repeated experiments in which statistical analysis is important. The main restriction of the technique is that it needs precisely timed activations (stimulus-triggered recordings) and recorded timecourses that can be explained by a response model. For slow

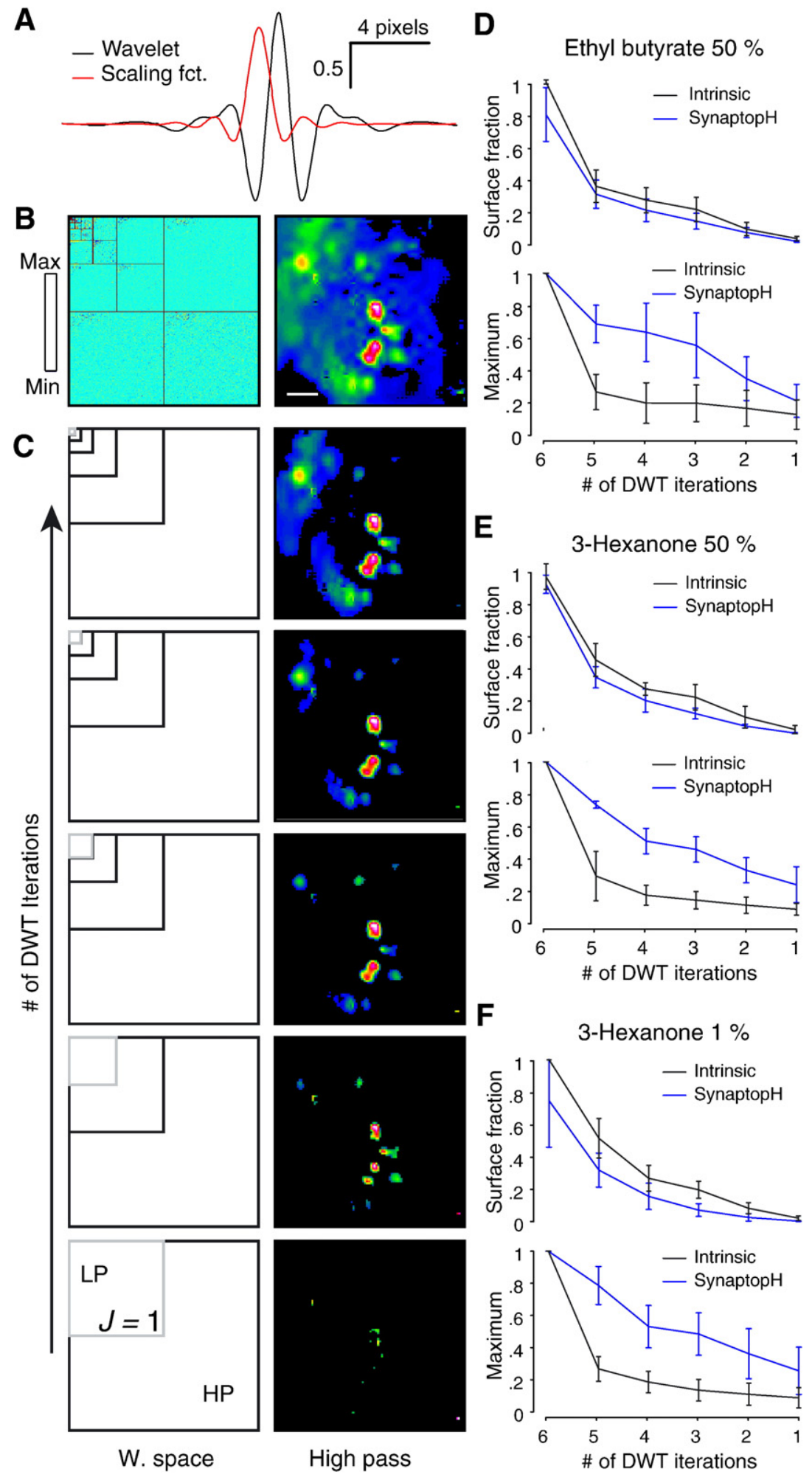


imaging, such as intrinsic signal, this condition is easily achieved. For fast imaging, such as voltage or calcium sensitive dyes imaging, the signal follows the rapid fluctuations of the neuronal activity, which can yield strong time-course heterogeneities among the pixels of a single recording or from experiment to experiment. For example, both voltage and calcium dye imaging in the olfactory bulb have revealed some temporal jitter between activations of different glomeruli (Spors and Grinvald, 2002; Spors et al., 2006). Unpredictable fluctuations or systematic timecourse variations are a problem for the LM fitting, but can be dealt with up to a certain extent. In general, artefact signals such as photobleaching are slow, and their regressor will not capture fast fluctuations that end up in the residual. Therefore, if the slow components of the relevant signal are well modelled, the time series can be reconstructed without information loss and without additional noise. However, it will not be possible to statistically assess fast components of the signal, and the significance will be reduced for slow components. In the case of systematic deviations, statistics can still be applied if one extends the LM by additional regressors that capture deviations from the idealized response (Friston et al., 1998). For example, glomeruli activation jitter can be captured by the partial derivative of the response model to the onset time, which would give (along with the global activation map) a statistically interpretable map of activation delays. Similarly, other type of artefact signals as described in this article could also be modelled (e.g. heart beat pulsation with the

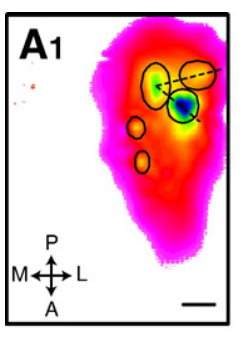

B1 Wavelet stat.
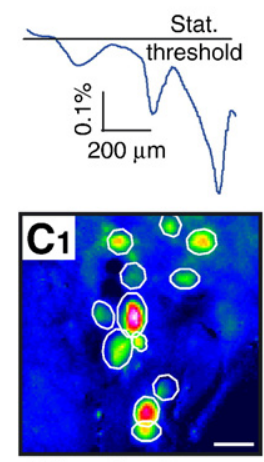

Wavelet \& stat.

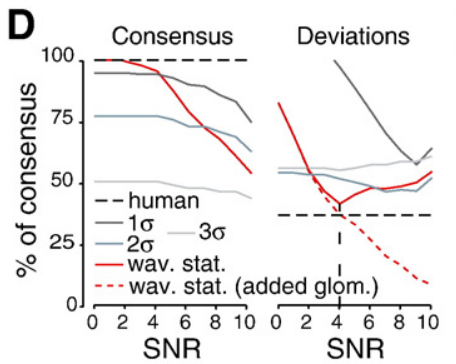

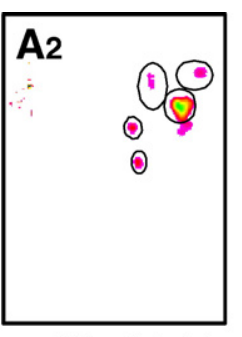

B2 Wavelet stat. + bandpass
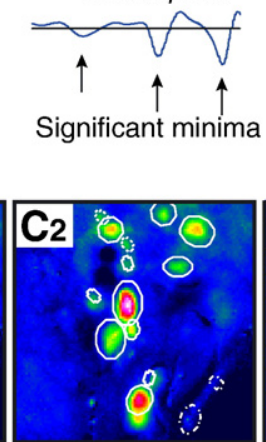

Gaussian $1 \sigma$

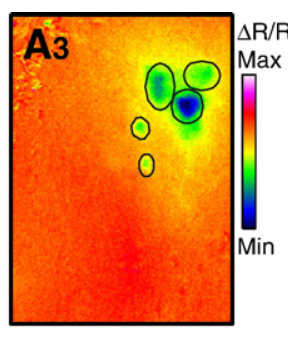

B3 Raw frame
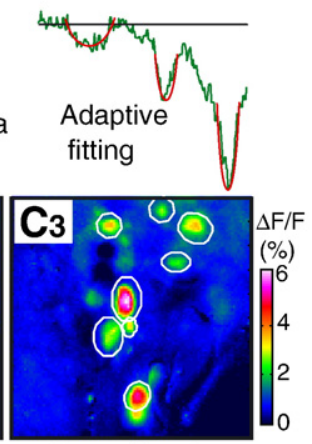

Gaussian $2 \sigma$
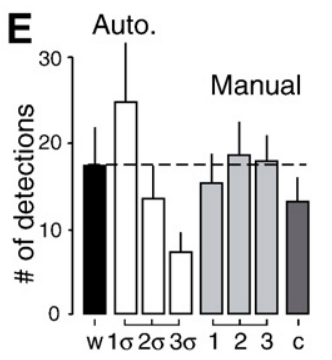

appropriate periodic function). In conclusion, while we exemplified the LM fitting framework for two applications in brain optical imaging only, we believe that it has a wide applicability to other types of optical recording. With appropriate regressors, fine tuned aspects (like activation jitters) of the signal can in principle be mapped and assessed independently.

\section{Assessing the significance of spatial details}

For the purpose of olfactory bulb physiology, we have developed a method to statistically assess activation patterns of a specific size. The method is based on wavelet sub-band selection to sort out details according to their size. Importantly, even when some wavelet coefficients are discarded, the statistics remain conservative; i.e. our method guarantees a strong type I error control (false positives) for "partial reconstructions" as well. We have demonstrated the usefulness of pattern assessment for glomeruli detection, but again, as the method is very general, it can also be applied to other biological contexts. Similar to the olfactory bulb, numerous brain areas are functionally (and anatomically) structured down to low spatial scales. In particular, most primary sensory cortical areas (at least somatosensory, visual and auditory areas) show spatial organization at the level of cortical columns (i.e. few hundreds micrometers). Optical imaging of the visual area V1, e.g. allows visualizing territories coding for retinal position (Grinvald et al., 1986; Schuett et al., 2002), orientation (Bonhoeffer and Grinvald, 1991), colour (Landisman and Ts'o, 2002) and left or right eye. These territories overlap but differ in their size and shape. Up to now, the different modalities have been independently studied. However, complex visual stimulation

Fig. 8. Comparison between manual and automated glomeruli detection. (A, B) Principle of glomeruli detection illustrated on the activation map (intrinsic signal, methylbenzoate $10 \%$ ) and the amplitude profile along the dashed line drawn in $\left(A_{1}\right) .\left(A_{1}, B_{1}\right)$. Full statistical evaluation of the activation. Colour bar: $-5 \%$ to $0 \%$. $\left(\mathrm{A}_{2}, \mathrm{~B}_{2}\right)$ Significant and consistent extrema are detected after high pass filtering and assessment. Colour bar: $-3.5 \%$ to $0 \%$. $\left(\mathrm{A}_{3}, \mathrm{~B}_{3}\right)$ Adaptive shape fitting and the raw image (LM treated image): a 2dimensional quadratic function is fitted to the local hill (or dip) surrounding the extremum to determine the extent of the glomerulus signal. Ellipsoids drawn in (A) represent the detections. Their size and orientation is determined by the fitting. Colour bar: $-5 \%$ to $1 \%$. Scale bar: $200 \mu \mathrm{m}$. (C) Automated detection for a $\mathrm{SpH}$ recording (amylacetate $10 \%$ was presented). Maxima are selected on the map of statistically assessed details $\left(\mathrm{C}_{1}\right)$, or on the frame average after band pass filtering and application of a fixed threshold $\left(\mathrm{C}_{2}: 1\right.$ image standard deviation; $\mathrm{C}_{3}: 2$ image standard deviation). For all three detections, a threshold of 4 is set on the signal to noise ratio of the fitted quadratic form. Discrepancies in the ellipsoid shapes are due to discrepancies in the localisation of the extremum. Scale bar: $200 \mu \mathrm{m}$. (D) Comparison of the automated detections (wavelet based statistical method and simple band pass filtering with 3 different thresholds: 1, 2 and 3 image standard deviations $\sigma$ ) to the consensus human detection (see text). Left. Average percentage of detected consensus regions as a function of signal to noise ratio (SNR) threshold. Right. Average percentage of detected non-consensus regions (sum of additional detections and missing detections) as a function of SNR threshold. The black dashed line represents the percentage of non-consensus detections for manual selection. The red dashed line represents the percentage of additional detections by the statistical method ( $n=10$ different odour presentations in 5 animals). (E) Average number of detections for automated and manual methods (w: wavelet statistical filtering, $1 \sigma, 2 \sigma, 3 \sigma$ : Gaussian band pass filtering and fixed thresholds, 1, 2, 3: individual human detections. c: consensus detections). Error bars (S.D.) represent the variability from odour to odour inside our data set. 
patterns are likely to produce complex activations in V1. In such case, multi-resolutions statistical analysis would help dissecting optical signals, and thus could be an interesting tool to analyse the spatial integration of different cortical processes. This type of analysis has also gained recent interest in human brain mapping (Breakspear et al., 2006). To conclude, we should mention that statistical analysis of activation patterns should not be restricted to optical imaging. The resolution of functional magnetic resonance imaging (fMRI) shows constant progress. Recently, fMRI odour maps (Xu et al., 2003, Schafer et al., 2006) have been recorded in the mouse and rat, opening the possibility of a totally non-invasive glomeruli mapping on the entire olfactory bulb. We have presented here a detection method designed for a $2 \mathrm{D}$ image, but clearly it would also be valid for a volume. Therefore a controlled detection of activated glomeruli in high-resolution fMRI images could be achieved with only slight modifications of our framework.

\section{Comparison between intrinsic signal and $\mathrm{SpH}$}

In this article, we have investigated two optical imaging techniques that were known to be indicative for olfactory bulb inputs, but which strongly differ in their mechanism. On the one hand, intrinsic signal is thought to rely on metabolic activity and/or cell swelling, which are both rather unspecific indicators of neural activity. On the other hand, $\mathrm{SpH}$ should be restricted by construction to nerve terminals and therefore to glomeruli. We found in fact that significantly activated areas were large for both $\mathrm{SpH}$ and intrinsic signal. However, a closer inspection of the activation captured in the individual wavelet sub-bands clearly showed that lowpass (unspecific) spatial components are small for $\mathrm{SpH}$ (and most likely comes from scattered and out of focus light) while they represents the majority of the signal amplitude for intrinsic imaging. This observation corroborates others (Meister and Bonhoeffer, 2001; Wachowiak and Cohen, 2003), suggesting that intrinsic signal originates from several sources in the olfactory bulb network. However, the precise identification of these sources has not been achieved yet, despite the potentially useful information it represents for OB physiology. As for glomeruli detection, it was clearly more difficult to find glomeruli activations in intrinsic signal images than in $\mathrm{SpH}$ images. The strong diffuse signal was the first obstacle to visual detection, but could be reduced by spatial high-pass filtering (similarly to Meister and Bonhoeffer, 2001). However, the signal-to-noise ratio after filtering was much higher than what was observed for $\mathrm{SpH}$ images, and thus the spatial definition of glomeruli was poorer. Moreover, noise had a substantial temporal correlation in intrinsic signal data, which was not observed in $\mathrm{SpH}$ recordings. Correlated noise is known to induce some bias on the linear model estimates that can affect the statistical evaluation. Probably, both higher noise amplitude and temporal correlation contributed to the much higher degree of uncertainty in the detections. In fact, for slightly subthreshold odorant concentrations, response patterns were generally sparse and glomeruli were well identifiable. In this case, our automated statistical detection method performed accurately (example Fig. 7A). However on a representative sample of odorant presentations, the level of non-consensus detections was as high as $80 \%$ for the human operator, while the statistical method could not do lower as $95 \%$ non-consensus detections. Therefore we concluded, in accordance with other studies (Wachowiak and Cohen, 2003), that intrinsic signal was sub-optimal for extensive glomeruli mapping.
In conclusion, highly specific techniques such as $\mathrm{SpH}$ imaging should be privileged over intrinsic imaging for extensive glomeruli identification. However, if some more knowledge is gained about the mechanisms of intrinsic signal, it might be very interesting to combine both techniques in the same experiment to try to obtain information about network activity on top of input patterns.

\section{How precise can extensive glomeruli detection be?}

The combination of statistical pattern assessment and basic post processing led us to automated glomeruli detection that performs as well as human operators. Importantly, the task proposed was to find, to the best extent, all the activated glomeruli in a dorsal OB recording. Extensive mapping of glomeruli is necessary to capture without bias the topographical rules that underlie the odour code. However, it is a difficult task, because if prominent activations are easy to identify as glomeruli, many zones (4 to 5 out of 17 on average) are doubtful and in fact subject to debate. By combining shape information with standard temporal activation profile, our statistical test secures that selected zones have the expected properties of an activated glomerulus. Interestingly, we found that the statistical test on itself detected more candidate glomeruli than the human operators ( $80 \%$ of the consensus compared to $35 \%$ ). That said, some of these zones were still very noisy or were obvious artefact due, e.g. to small heterogeneities in the tissue or in the bone that scattered fluorescent light. We could remove such zones with a simple post-processing method that probes the regularity of the spatial activation profile. In all cases, technical limitations are still potential sources of error in the detection. Glomeruli are variable in size, but their density is also rather heterogeneous and their arrangement is not strictly bi-dimensional. It is a common observation in olfactory bulb slices that a glomerulus can sit on top of another one, rendering their separation virtually impossible by optical imaging. Therefore, while the present detection method is able to localize the majority of activations, this technical difficulty might limit a complete mapping of the glomeruli. The use of other imaging techniques, such as two-photon microscopy (Wachowiak et al., 2004), could further improve these results.

\section{A framework for more precision in the study of olfactory bulb inputs}

Lastly, it is interesting to ask what may be the impact of our methods on the study of olfactory bulb physiology. LM fitting yielded a substantial reduction of the noise and improved reproducibility of response maps (Fig. 4). In addition, we observed in many cases that the LM fitting procedure strongly attenuated some occasional perturbations which we had not included in the models (e.g. signal from blood vessels; see Supplementary Fig. 1). Although treating such perturbations was not the goal of our approach, we see the robust behaviour of the LM as another evidence for its efficiency. Based on the LM, wavelet statistical filtering provides a very good method for noise rejection (optimal smoothing-Fig. 6). But probably the most valuable contribution is brought by the multi-resolution statistical analysis. By selecting the wavelet sub-band corresponding to the typical size of glomeruli, one can well isolate the signal specific of these structures. Moreover, because the statistical analysis still 
applies to sub-band selection, the framework provides a conservative method to decide whether a glomerulus is active given the level of noise in the recordings. The performance of the framework can be illustrated if one compares concentrationresponse curves of glomeruli built after LM fitting and wavelet sub-band selection with curves obtained with the standard methods (Supplementary Fig. 2). On the whole, the behaviour of glomeruli response seems to be unchanged for higher concentrations, although the absolute amplitude of the response might be decreased because of the filtering (i.e. sub-band selection) which removes large scale signals components that are not really specific to glomeruli. At lower concentration, the wavelet framework greatly regularizes the concentration-response curve (Supplementary Fig. 2C). Indeed, filtering and statistical threshold has removed non-relevant (e.g. spatially too extended to be from glomeruli) and non-significant components of the signal. On the other hand, the method is sensitive enough to detect glomeruli with a low activation, which may be hardly detectable on standard images, but then become clear when concentration is increased. For example in Figs. 6B and A (methyl benzoate 1\% and $20 \%$ ), the bottom right activation in Fig. $6 \mathrm{~B}_{2}$ is hardly seen in Fig. $6 \mathrm{~B}_{1}$ but then clearly appears in Fig. 6A (see also glomeruli 4 and 5 from $5 \%$ to $20 \%$ concentration in Supplementary Figs. 2A and B). Altogether, these observations indicate that our statistical framework has a large potential for improvement of robustness and precision in olfactory bulb inputs imaging.

\section{Acknowledgments}

We thank C. Petersen and R. Accolla for comments on the manuscript. This work was supported by the Brain Mind Institute, Ecole Polytechnique Fédérale de Lausanne (A.C.), by the Center for Biomedical Imaging (CIBM) of the Geneva, Lausanne Universities and the EPFL, as well as the foundations Leenaards and Louis-Jeantet (D.V.; T.B.; M.U.).

\section{Appendix A. Supplementary data}

Supplementary data associated with this article can be found, in the online version, at doi:10.1016/j.neuroimage.2006.10.038.

\section{References}

Abraham, N.M., Spors, H., Carleton, A., Margrie, T.W., Kuner, T., Schaefer, A.T., 2004. Maintaining accuracy at the expense of speed; stimulus similarity defines odor discrimination time in mice. Neuron 44, 865-876.

Belluscio, L., Katz, L.C., 2001. Symmetry, stereotypy, and topography of odorant representations in mouse olfactory bulbs. J. Neurosci. 21, 2113-2122.

Bonhoeffer, T., Grinvald, A., 1991. Iso-orientation domains in cat visual cortex are arranged in pinwheel-like patterns. Nature 353, 429-431.

Bozza, T., McGann, J.P., Mombaerts, P., Wachowiak, M., 2004. In vivo imaging of neuronal activity by targeted expression of a genetically encoded probe in the mouse. Neuron 42, 9-21.

Breakspear, M., Bullmore, E.T., Aquino, K., Das, P., Williams, L.M., 2006. The multiscale character of evoked cortical activity. NeuroImage 30, $1230-1242$.

Bullmore, E., Fadili, J., Breakspear, M., Salvador, R., Suckling, J., Brammer, M., 2003. Wavelets and statistical analysis of functional magnetic resonance images of the human brain. Stat. Methods Med. Res. 12, 375-399.

Djurisic, M., Antic, S., Chen, W.R., Zecevic, D., 2004. Voltage imaging from dendrites of mitral cells: EPSP attenuation and spike trigger zones. J. Neurosci. 24, 6703-6714.

Fadili, M.J., Bullmore, E.T., 2004. A comparative evaluation of waveletbased methods for hypothesis testing of brain activation maps. NeuroImage 23, 1112-1128.

Friston, K.J., Fletcher, P., Josephs, O., Holmes, A., Rugg, M.D., Turner, R., 1998. Event-related fMRI: characterizing differential responses. NeuroImage 7, 30-40.

Frostig, R.D., Lieke, E.E., Ts'o, D.Y., Grinvald, A., 1990. Cortical functional architecture and local coupling between neuronal activity and the microcirculation revealed by in vivo high-resolution optical imaging of intrinsic signals. Proc. Natl. Acad. Sci. U. S. A. 87, 6082-6086.

Grinvald, A., Hildesheim, R., 2004. VSDI: a new era in functional imaging of cortical dynamics. Nat. Rev., Neurosci. 5, 874-885.

Grinvald, A., Lieke, E., Frostig, R.D., Gilbert, C.D., Wiesel, T.N., 1986. Functional architecture of cortex revealed by optical imaging of intrinsic signals. Nature 324, 361-364.

Grinvald, A., Shoham, D., Shmuel, A., Glaser, D.E., Vanzetta, I., Shtoyerman, E., Slovin, H., Wijnbergen, C., Hildesheim, R., Sterkin, A., Arieli, A., 1999. In-vivo optical imaging of cortical architecture and dynamics. In: Windhorst, U., Johansson, H. (Eds.), Modern Techniques in Neuroscience Research. Springer Verlag, pp. 893-969.

Hasan, M.T., Friedrich, R.W., Euler, T., Larkum, M.E., Giese, G., Both, M., Duebel, J., Waters, J., Bujard, H., Griesbeck, O., et al., 2004. Functional fluorescent $\mathrm{Ca} 2+$ indicator proteins in transgenic mice under TET control. PLoS Biol. 2, e163.

Landisman, C.E., Ts'o, D.Y., 2002. Color processing in macaque striate cortex: relationships to ocular dominance, cytochrome oxidase, and orientation. J. Neurophysiol. 87, 3126-3137.

Mallat, S., 1989. A theory for multiresolution signal decomposition: the wavelet decomposition. IEEE Trans. Pattern. Anal. Mach. Intell. 11, 674-693.

Mallat, S., 1999. A Wavelet Tour of Signal Processing. Academic Press.

McGann, J.P., Pírez, N., Gainey, G.A., Muratore, C., Elias, A.S., Wachowiak, M., 2005. Odorant representations are modulated by intra- but not interglomerular presynaptic inhibition of olfactory sensory neurons. Neuron 48, 1039-1053.

Meister, M., Bonhoeffer, T., 2001. Tuning and topography in an odor map on the rat olfactory bulb. J. Neurosci. 21, 1351-1360.

Miesenbock, G., De Angelis, D.A., Rothman, J.E., 1998. Visualizing secretion and synaptic transmission with $\mathrm{pH}$-sensitive green fluorescent proteins. Nature 394, 192-195.

Mombaerts, P., 2004. Genes and ligands for odorant, vomeronasal and taste receptors. Nat. Rev., Neurosci. 5, 263-278.

Rubin, B.D., Katz, L.C., 1999. Optical imaging of odorant representations in the mammalian olfactory bulb. Neuron 23, 499-511.

Ruttimann, U.E., Unser, M., Rawlings, R.R., Rio, D., Ramsey, N.F., Mattay, V.S., Hommer, D.W., Frank, J.A., Weinberger, D.R., 1998. Statistical analysis of functional MRI data in the wavelet domain. IEEE Trans. Med. Imaging 17, 142-154.

Schafer, J.R., Kida, I., Xu, F., Rothman, D.L., Hyder, F., 2006 Reproducibility of odor maps by fMRI in rodents. NeuroImage 31, 1238-1246.

Schuett, S., Bonhoeffer, T., Hubener, M., 2002. Mapping retinotopic structure in mouse visual cortex with optical imaging. J. Neurosci. 22, $6549-6559$.

Spors, H., Grinvald, A., 2002. Spatio-temporal dynamics of odor representations in the mammalian olfactory bulb. Neuron 34, 301-315.

Spors, H., Wachowiak, M., Cohen, L.B., Friedrich, R.W., 2006. Temporal dynamics and latency patterns of receptor neuron input to the olfactory bulb. J. Neurosci. 26, 1247-1259.

Turkheimer, F.E., Brett, M., Aston, J.A., Leff, A.P., Sargent, P.A., Wise, R.J., Grasby, P.M., Cunningham, V.J., 2000. Statistical modeling of positron 
emission tomography images in wavelet space. J. Cereb. Blood Flow Metab. 20, 1610-1618.

Uchida, N., Mainen, Z.F., 2003. Speed and accuracy of olfactory discrimination in the rat. Nat. Neurosci. 6, 1224-1229.

Van De Ville, D., Blu, T., Unser, M., 2003. Wavelets versus resels in the context of fMRI: establishing the Link with SPM. Paper presented at: SPIE Conference on Mathematical Imaging: Wavelet Applications in Signal and Image Processing X (San Diego CA, USA).

Van De Ville, D., Blu, T., Unser, M., 2004. Integrated wavelet processing and spatial statistical testing of fMRI data. NeuroImage 23, 1472-1485.

Van De Ville, D., Blu, T., Unser, M., 2006. Surfing the brain. IEEE Eng. Med. Biol. Mag. 25, 65-78.
Wachowiak, M., Cohen, L.B., 2001. Representation of odorants by receptor neuron input to the mouse olfactory bulb. Neuron 32, 723-735.

Wachowiak, M., Cohen, L.B., 2003. Correspondence between odorantevoked patterns of receptor neuron input and intrinsic optical signals in the mouse olfactory bulb. J. Neurophysiol. 89, 1623-1639.

Wachowiak, M., Denk, W., Friedrich, R.W., 2004. Functional organization of sensory input to the olfactory bulb glomerulus analyzed by two-photon calcium imaging. Proc. Natl. Acad. Sci. U. S. A. 101, 9097-9102.

Xu, F., Liu, N., Kida, I., Rothman, D.L., Hyder, F., Shepherd, G.M., 2003. Odor maps of aldehydes and esters revealed by functional MRI in the glomerular layer of the mouse olfactory bulb. Proc. Natl. Acad. Sci. U. S. A. 100, 11029-11034. 